\title{
ON THE OSCILLATION OF SOLUTIONS OF SELF- ADJOINT LINEAR DIFFERENTIAL EQUATIONS OF THE FOURTH ORDER
}

\author{
BY \\ WALTER LEIGHTON AND ZEEV NEHARI(1) \\ Dedicated to Professor Marston Morse
}

1. Introductory remarks. The present paper is devoted to a more or less systematic investigation of the oscillation properties of the solutions of certain classes of self-adjoint linear differential equations of fourth order. The most general equation of this type is of the form

$$
\left(r(x) y^{\prime \prime}\right)^{\prime \prime}+\left(q(x) y^{\prime}\right)^{\prime}+P(x) y=0, \quad r(x)>0,
$$

where, in the interval considered, $r(x), q(x)$ and $P(x)$ are functions of $C^{\prime \prime}$, $C^{\prime}$ and $C$, respectively, and the prime denotes differentiation with respect to $x$. Unless there is an explicit statement to the contrary, it will be assumed that all these functions are defined, and belong to the classes indicated, in the interval $(0, \infty)$.

Simple examples show that the general equation (1.1) includes equations of radically different oscillatory behavior. For a more penetrating study of the solutions of (1.1) it is therefore necessary to introduce distinctions between certain sub-classes of equations (1.1) which reflect these basic differences. Taking our cue from the analogous second-order equation

$$
\left(r(x) y^{\prime}\right)^{\prime}+P(x) y=0
$$

whose oscillatory behavior is very different according as $P(x)$ is positive or negative, we shall-in the case $q(x)=0$, i.e. in the case of the equation

$$
\left(r(x) y^{\prime \prime}\right)^{\prime \prime}+P(x) y=0,
$$$$
r(x)>0
$$

- distinguish between two cases, according as $P(x)$ is positive or negative in $(0, \infty)$. In order to express this distinction in our notation we shall write these equations in the form

$$
\left(r(x) y^{\prime \prime}\right)^{\prime \prime}-p(x) y=0,
$$

$$
r(x)>0, p(x)>0,
$$

and

$$
\left(r(x) y^{\prime \prime}\right)^{\prime \prime}+p(x) y=0,
$$$$
r(x)>0, p(x)>0 .
$$

We shall not discuss the case in which $P(x)$ may change its sign in $(0, \infty)$.

Presented to the Society December 29, 1956; received by the editors March 18, 1957.

(1) This research was supported by the United States Air Force Office of Scientific Research. 
As in the case of the equation (1.2), such a change of sign causes a profound change in the character of the equation, and one is almost tempted to regard the zeros of $P(x)$ as singularities of the equation, at least as far as oscillatory behavior is concerned.

We shall confine our discussion to equations (1.4) and (1.5) and to such equations (1.1) which can be transformed into one or the other of these types. There exist a number of transformations which will remove th term of equation (1.1) if $q(x)$ satisfies certain conditions, and some of these transformations will be discussed in $\$ 12$.

A remark has to be made here about our use of the term "oscillatory." As customary, we shall call a solution oscillatory if it has an infinite number of zeros in $(0, \infty)$ and we shall refer to it as nonoscillatory if this is not the case. An equation is termed nonoscillatory if all its solutions are nonoscillatory, and it is called oscillatory if at least one of its solutions is oscillatory. The latter definition is made necessary by the fact that it may very well happen that some of the solutions of an equation (1.3) are oscillatory while some others are not. Indeed, we shall see that one of the features which set equations (1.4) and (1.5) apart is the fact that (1.4) has always some nonoscillatory solutions, while the solutions of (1.5) are either all oscillatory or all nonoscillatory.

The equation (1.2) is often said to be "nonoscillatory in $(a, \infty)$ " if none of its solutions has more than one zero in this interval. We shall use the same statement with reference to the equation (1.1) if none of its solutions has more than three zeros in $(a, \infty)$. This is in the nature of things, since the equation is easily shown to have solutions with zeros at three arbitrarily given points. If (1.2) is nonoscillatory then, because of the Sturm separation theorem, there will exist a value $a$ such that the equation is nonoscillatory in $(a, \infty)$. The corresponding fact for the equation (1.3) seems to be of a less elementary character and we shall prove it separately for the equations (1.4) and (1.5).

Part I ( $\S 2-7)$ of this paper will be devoted to the study of the solutions of equation (1.4), and the investigation of the solutions of (1.5) will be taken up in Part II ( $\S 8-11)$. In both cases we are mainly concerned with topics familiar from the theory of the equation (1.2), namely, separation and comparison theorems and oscillation criteria. As may be expected, however, there will also appear phenomena which have no analogue in the second-order case. The study of equation (1.4) is, of course, closely related to that of the eigenvalue problems arising in the theory of the vibrating rod [3]. In $\$ 4$ we shall, accordingly, devote some space to a discussion of those aspects of these eigenvalue problems which have a bearing on our main topic.

The investigation of the oscillation properties of linear differential equations of order higher than the second was given its impetus by a classical paper of G. D. Birkhoff on the third-order equation [1]. The fourth-order 
case has been explored by a number of authors $[2 ; 11 ; 12 ; 13 ; 14]$. As regards both methods and results, there are few, if any, points of contact between this earlier work and the present paper. A similar remark applies to the work of Marston Morse on systems of second-order equations [9, especially Chapter IV], although there appear to be certain connections between this work and some of the developments in $\S 3$ which deserve further study.

In the discussion of both (1.4) and (1.5) we shall repeatedly have occasion to make use of an elementary argument which, for the sake of convenience, we shall state as a lemma.

Lemma 1.1. Let $u(x)$ and $v(x)$ be of class $C^{\prime}$ in $(a, b)$ and let $v(x)$ be of constant sign in this interval. If $u(x)$ has two distinct zeros in $(a, b)$, then the function $\sigma(x)=v(x) u^{\prime}(x)-u(x) v^{\prime}(x)$ cannot be of constant sign in the interval bounded by these zeros.

Indeed, suppose that $x=\alpha$ and $x=\beta$ are consecutive zeros of $u(x)$ $(a<\alpha<\beta<b)$. If, say, $\sigma(x)>0$ in $(\alpha, \beta)$, then $0=u(\beta) v^{-1}(\beta)=\int_{\alpha}^{\beta} \sigma v^{-2} d x>0$, and this contradiction proves the lemma.

For some applications it is more convenient to state this lemma in the following form.

Lemma 1.2. Let $u(x)$ and $v(x)$ be of class $C^{\prime}$ in $(a, b)$ and let $v(x)$ be of constant sign in this interval. If $x=\alpha$ and $x=\beta(a<\alpha<\beta<b)$ are consecutive zeros of $u(x)$, then there exists a constant $\mu$ such that the function $u(x)-\mu v(x)$ has a double zero in $(\alpha, \beta)$.

By a double zero we mean, as usual, that both the function and its derivative vanish at the point in question. The equivalence of Lemma 1.1 and Lemma 1.2 follows from the remark that $\sigma\left(x_{0}\right)=0$ is equivalent to the existence of two constants $\lambda, \mu\left(\lambda^{2}+\mu^{2}>0\right)$ such that $\lambda u\left(x_{0}\right)-\mu v\left(x_{0}\right)=\lambda u^{\prime}\left(x_{0}\right)$ $-\mu v^{\prime}\left(x_{0}\right)=0$. Since $v(x) \neq 0$ in $[\alpha, \beta], \lambda$ cannot be zero and may therefore be taken to be unity.

\section{PART I}

2. General properties of solutions of (1.4).

1. We begin the discussion of the equation

$$
\left(r(x) y^{\prime \prime}\right)^{\prime \prime}-p(x) y=0, \quad r(x)>0, p(x)>0,
$$

by proving the following result.

LemMa 2.1. If $y(x)$ is a solution of (2.1) and the values of $y, y^{\prime}, y^{\prime \prime}$ and $\left(r y^{\prime \prime}\right)^{\prime}$ are non-negative (but not all zero) for $x=a$, then the functions $y(x), y^{\prime}(x), y^{\prime \prime}(x)$ and $\left[r(x) y^{\prime \prime}(x)\right]^{\prime}$ are positive for $x>a$.

The case in which all the initial values are zero must, of course, be excluded since by the existence theorem $y(x)$ would then reduce to the trivial solution $y(x)=0$. To prove the lemma we observe that in view of our assump- 
tions we have either $y(a)>0$, or else the first nonvanishing derivative of $y(x)$ at $x=a$ is positive. In all cases $y(x)$ will therefore be positive in some open interval whose left boundary point is $x=a$. If $y(x)$ is not positive for all $x$ such that $x>a$, there will exist a point $x=x_{0}$ such that $y\left(x_{0}\right)=0$ and $y(x)>0$ for $a<x<x_{0}$.

It follows from (2.1) that

$$
r(x) y^{\prime \prime}(x)=\left[r y^{\prime \prime}\right]_{a}+(x-a)\left[\left(r y^{\prime \prime}\right)^{\prime}\right\rfloor_{a}+\int_{a}^{x}(x-t) p(t) y(t) d t .
$$

Evidently, the right-hand side is positive, and increases with $x$, as long as $y(x)$ remains positive. We thus conclude that $y^{\prime \prime}(x)$ and $\left[r(x) y^{\prime \prime}(x)\right]^{\prime}$ are positive for $a<x<x_{0}$. Since $y^{\prime}(a) \geqq 0, y^{\prime}(x)$ must likewise be positive in this interval, and we arrive at the contradiction $0 \leqq y(a)<y\left(x_{0}\right)=0$. This proves that $y(x)$-and also $y^{\prime}(x), y^{\prime \prime}(x),\left[r(x) y^{\prime \prime}(x)\right]^{\prime}$-remain positive for all $x>a$.

Lemma 2.1 shows that equation (2.1) always has some nonoscillatory solutions and it also indicates how such solutions may be generated.

It is easy to convert Lemma 2.1 into a result concerning the behavior of certain solutions of (2.1) at points $x$ such that $x<a$. If $c$ is a positive number, the substitution $x=a+c-t$ will transform (2.1) into a differential equation of the same type in the independent variable $t$. An application of Lemma 2.1 at the point $t=c$ leads to the following result.

LeMma 2.2. Let $y(x)$ be a nontrivial solution of (2.1) and let a be positive. If $y(a) \geqq 0, y^{\prime \prime}(a) \geqq 0, y^{\prime}(a) \leqq 0$ and $\left[r y^{\prime \prime}\right]_{a}^{\prime} \leqq 0$, then $y(x)$ and $y^{\prime \prime}(x)$ are positive, and $y^{\prime}(x)$ and $\left[r(x) y^{\prime \prime}(x)\right]^{\prime}$ are negative for $0<x<a$.

We are now ready to prove a result which will play a fundamental role in the sequel.

Lemma 2.3. Let $y(x)$ be a nontrivial solution of (2.1) and let $a, b, c$ be values such that $0<a<b<c$. If $y(a)=y(b)=y(c)=0$, then $y^{\prime}(b) \neq 0$.

Indeed, suppose $y(b)=y^{\prime}(b)=0$. Without loss of generality, we may assume that $y^{\prime \prime}(b) \geqq 0$. We now consider the quantity $A=\left[\left(r y^{\prime \prime}\right)^{\prime}\right]_{x=b}$. If $A \geqq 0$, it follows from Lemma 2.1 that $y(x)>0$ for $x>a$, and this contradicts the assumption $y(c)=0$. Similarly, $A \leqq 0$ conflicts with Lemma 2.2 and the assumption $y(a)=0$, and our lemma is proved.

Since under the assumptions of Lemma 2.1 none of the quantities $y, y^{\prime}$, $y^{\prime \prime},\left(r y^{\prime \prime}\right)^{\prime}$ can be zero for $x>a$, Lemma 2.3 may be extended to the following more general statement.

Lemma 2.4. Let $y(x)$ be a nontrivial solution of (2.1) and let $a, b, c$ be such that $0<a<b<c$. If $y(b)=y^{\prime}(b)=0$, then in at least one of the two intervals $(0, b)$, $(b, \infty)$ all of the functions $y, y^{\prime}, y^{\prime \prime},\left(r y^{\prime \prime}\right)^{\prime}$ are different from zero.

Of the other possible generalizations we confine ourselves to the following result whose proof is essentially the same as that of Lemma 2.3. 
LEMмA 2.5. The conclusion of Lemma 2.4 remains valid if the condition $y(b)=y^{\prime}(b)=0$ is replaced by $y^{\prime \prime}(b)=y^{\prime \prime \prime}(b)=0$.

2. As already mentioned in $\$ 1$, there always exist nontrivial solutions of (1.1) which vanish in three given (not necessarily distinct) points. Indeed, the general solution of (1.1) is of the form $y(x)=\sum_{n=1}^{4} a_{n} y_{n}(x)$, where $\left\{y_{n}(x)\right\}$ is a fundamental system and the $a_{n}$ are constants, and the conditions $y(\alpha)$ $=y(\beta)=y(\gamma)=0$ lead to a system of linear equations for the $a_{n}$ which always has a nontrivial solution. In general there may be even two or more essentially different solutions of (1.1) which have the same three zeros in common (two solutions will be called "essentially different" if they are not constant multiples of each other). It may even happen that two essentially different solutions have an infinity of zeros in common, as is shown by the equation $y^{\mathrm{iv}}+4 y=0$ which has the solutions $y_{1}(x)=\sin x \sinh x$ and $y_{2}(x)=\sin x \cosh x$. It is therefore remarkable that the following uniqueness theorem holds for the solutions of (2.1).

THEOREM 2.6. If two nontrivial solutions of (2.1) have three zeros in common, then they are constant multiples of each other.

We first suppose that the three zeros, say $a, b, c$, are distinct $(0<a<b<c)$. If $u(x)$ and $v(x)$ are the two solutions in question then, by Lemma 2.3, $u^{\prime}(b) \neq 0$ and $v^{\prime}(b) \neq 0$. In view of the assumptions about $u(x)$ and $v(x)$, the solution $w(x)=v^{\prime}(b) u(x)-u^{\prime}(b) v(x)$ will thus satisfy $w(a)=w(b)=w^{\prime}(b)=w(c)$ $=0$. But, again by Lemma 2.3 , this is impossible unless $w(x) \equiv 0$, that is, unless $v(x)$ is a constant multiple of $u(x)$. If two of these zeros coincide, say $a=b$, we consider $w(x)=v^{\prime \prime}(a) u(x)-u^{\prime \prime}(a) v(x)$ instead. Both $u^{\prime \prime}(a)$ and $v^{\prime \prime}(a)$ must be different from zero since otherwise it would follow from Lemma 2.1 that $u(x)$ or $v(x)$ could not vanish at $x=c$. But the solution $w(x)$ would have a triple zero at $x=a$ and another zero at $x=c$, and this again contradicts Lemma 2.1 unless $w(x)=0$. Finally, two nontrivial solutions with a common triple zero are essentially identical since, according to the existence theorem for the equation (1.1), a solution is determined uniquely by the conditions $y(a)$ $=y^{\prime}(a)=y^{\prime \prime}(a)=0, y^{\prime \prime \prime}(a)=1$. This completes the proof of Theorem 2.6.

\section{Separation theorems.}

1. Simple examples show that the zeros of two solutions of (2.1) do not necessarily separate each other in the same regular fashion as the zeros of two solutions of (1.2). This is not.surprising, since three zeros of a solution of (2.1) may be given arbitrarily, and between two consecutive zeros of one solution there may therefore lie three zeros of another solution. However, if account is taken of this and some other peculiarities of the fourth-order case, a considerable measure of regularity appears.

Before we enter into the discussion of specific separation theorems, we define a coneept which plays an important-although not always explicitrole in the separation and oscillation theory of (2.1). Let $y(x)$ be a solution of 
(2.1) for which $y(a)=0$ and suppose that $y(x)$ has at least $n+3$ zeros $a_{1}$, $a_{2}, \cdots, a_{n+3}\left(a=a_{1} \leqq a_{2} \leqq \cdots \leqq a_{n+3}, n \geqq 1\right)$ for $x \geqq a$ (it may be noted that the sign of equality cannot hold more than twice since, by virtue of Lemma 2.3 , no solution of (2.1) can have more than two double zeros; moreover, if a solution has two double zeros, then all its zeros are confined to the closed interval bounded by the double zeros). We now define the $n$-th conjugate point of $a$ (cf. [9]) as the minimum value of $a_{n+3}$ as $y(x)$ ranges over all solutions of (2.1) for which $y(a)=0$. If there exists at least one solution $y(x)$ with the requisite number of zeros in $(a, \infty)$, then the existence of the $n$-th conjugate point is easily established with the help of a compactness argument. This will, however, not be necessary since we shall obtain the conjugate points by direct construction.

2. Our first separation theorem deals with a case in which there is simple separation of the Sturm type.

THEOREM 3.1. If $u(x)$ and $v(x)$ are two essentially different nontrivial solutions of (2.1) such that $u(a)=v(a)=u(b)=v(b)=0(0<a<b)$, then the zeros of $u(x)$ and $v(x)$ in $(a, b)$ separate each other.

We remark that the theorem is not empty since there always exist essentially different solutions which have the zeros $x=a, b$ in common. Indeed, if $u(x)$ is one such solution, we may take $v(x)$ to be the solution characterized by the conditions $v(a)=v(b)=v(c)=0$, where $c$ is such that $u(c) \neq 0$. To prove the theorem, we remark that, by Theorem 2.6 , no zero of $u(x)$ in $(a, b)$ can coincide with a zero of $v(x)$. If the conclusion of the theorem were not true, there would then exist two consecutive zeros $x=\alpha, \beta(a<\alpha<\beta<b)$ of one of the solutions, say $u(x)$, such that $v(x) \neq 0$ in $[\alpha, \beta]$. By Lemma 1.2, there would thus exist a constant $\mu$ such that $w(x)=u(x)-\mu v(x)$ has a double zero in $(\alpha, \beta)$. Since $w(x)$ is a solution of $(2.1)$ and $w(a)=w(b)=0$, this contradicts Lemma 2.3, and the proof of Theorem 3.1 follows.

Simple examples show that the first and the last zeros in $(a, b)$ may both belong to the same solution. We are therefore unable to conclude that $u(x)$ and $v(x)$ have the same number of zeros in $(a, b)$ and we must content ourselves with the following statement.

CoROllary 3.2. If two nontrivial solutions $u(x)$ and $v(x)$ of $(2.1)$ have the two zeros $x=a, b$ in common, then the number of zeros of $u(x)$ in $(a, b)$ differs from the number of zeros of $v(x)$ in $(a, b)$ at most by 1 .

The assumption of two common zeros under which Theorem 3.1 was proved is rather restrictive. However, some condition of this kind is clearly required in order to compare the number of zeros of two solutions. Lemma 2.1 shows that a given solution of (2.1) may have an arbitrary number of zeros in an interval in which other solutions of (2.1) do not vanish at all. As the following result indicates, this possibility is excluded by the assumption that the solutions in question have zeros beyond the interval considered. 
THEOREM 3.3. Let $u(x)$ and $v(x)$ be two nontrivial solutions of (2.1) which vanish at $x=a$. If $u(b)=v(c)=0(a<b<c)$ and $n$ and $m$ denote the number of zeros of $u(x)$ and $v(x)$, respectively, in $(a, b]$, then $n-2 \leqq m \leqq n+2$.

We take $x=c$ to be the smallest zero of $v(x)$ in $(b, \infty)$, and introduce the (by Theorem 2.6 essentially unique) solution $w(x)$ characterized by the conditions $w(a)=w(b)=w(c)=0$. Theorem 3.1 may then be applied to the solutions $u(x)$ and $w(x)$ in $(a, b)$ and to the solutions $v(x)$ and $w(x)$ in $(a, c)$, respectively, and the result follows. It may also be noted that the existence of a solution $w(x)$ with the indicated properties implies the following substitute for a separation theorem.

THEOREM 3.4. Let $u(x)$ and $v(x)$ be two nontrivial solutions of (2.1) which vanish at $x=a$. If $u(b)=v(c)=0(a<b<c)$, there exists another solution of (2.1) which vanishes at $x=a$ and whose zeros in $(a, b)$ separate both the zeros of $u(x)$ and those of $v(x)$ in this interval.

The same principle of proof may also be applied to the more general case in which $u(a)=u(b)=0, v\left(a^{\prime}\right)=v\left(b^{\prime}\right)=0$ and $0<a^{\prime}<a<b<b^{\prime}$. By constructing solutions $w(x)$ with zeros at $a^{\prime}, a, b$ and $a, b, b^{\prime}$, respectively, and applying Theorems 3.1 and 3.3 , we obtain the following result.

THEOREM 3.5. Let $u(x)$ and $v(x)$ be two nontrivial solutions of (2.1) for which $u(a)=u(b)=v\left(a^{\prime}\right)=v\left(b^{\prime}\right)=0$, where $0<a^{\prime}<a<b<b^{\prime}$. If $n$ and $m$ denote the number of zeros in $[a, b]$ of $u(x)$ and $v(x)$, respectively, then $n-3 \leqq m \leqq n+3$.

3 . We are now ready to discuss the extremal problem leading to the notion of the $n$-th conjugate points introduced above. Consider the solutions $y(x)$ of (2.1) which vanish at $x=a$ and-assuming this class is not empty-have at least $n+3$ zeros in $[a, \infty)$, where $n$ is a given positive integer. We denote by $a_{1}, a_{2}, \cdots, a_{n+3}\left(a_{1}=a\right)$ the first $n+3$ zeros of $y(x)$ in $[a, \infty)$, and we seek an extremal solution which minimizes the value of $a_{n+3}$ in this class. Corollary 3.2 shows that we may restrict ourselves to the class of solutions which have a double zero at $x=a$. Indeed, if $y(x)$ is the solution just mentioned and if $a_{1}<a_{2}$, we may compare the number of its zeros in $\left(a, a_{n+3}\right)$ with that of the solution $v(x)$ for which $v(a)=v^{\prime}(a)=v\left(a_{n+3}\right)=0$. The extra zero which $y(x)$ may have in $\left(a, a_{n+3}\right)$ according to Corollary 3.2 is made up for by the double zero of $v(x)$ at $x=a$ (multiple zeros are to be counted according to their multiplicities), and $v(x)$ has therefore at least as many zeros in $\left[a, a_{n+3}\right]$ as $y(x)$.

We again denote by $a_{1}, a_{2}, \cdots, a_{n+3}\left(a=a_{1}=a_{2}\right)$ the first $n+3$ zeros of $v(x)$ in $[a, \infty)$, and we introduce a solution $w(x)$ of $(2.1)$ which has a double zero at $x=a$ and is positive for $x>a$. According to Lemma 2.1, we may, for instance, take the solution $w(x)$ determined by the conditions $w(a)=w^{\prime}(a)$ $=w^{\prime \prime \prime}(a)=0, w^{\prime \prime}(a)=1$. If $a_{n+2}$ does not coincide with $a_{n+3}$, we may conclude from Lemma 1.2 that there exists a constant $\mu$ such that the solution $w_{1}(x)$ 
$=v(x)-\mu w(x)$ has a double zero in the interval $\left(a_{n+2}, a_{n+3}\right)$, say at the point $x=\alpha\left(a_{n+2}<\alpha<a_{n+3}\right)$. The function $w_{1}(x)$ is the (essentially) unique solution of our extremal problem. To prove this assertion, we assume without loss in generality that $v(x)>0$ in $\left(a_{n+2}, a_{n+3}\right)$. It is then easy to see that the curve $\xi=\mu w(x)$ has precisely two points of intersection with every positive arch of the curve $\xi=v(x)$ which is located in $\left(a_{3}, a_{n+2}\right)$. Indeed, since $v(\alpha)-\mu w(\alpha)=0$ and both $v(\alpha)$ and $w(\alpha)$ are positive, the constant $\mu$ must be positive. If there existed a positive arch of $\xi=v(x)$ in $\left(a_{3}, a_{n+2}\right)$ which is not intersected by $\xi=\mu w(x)$, there would then, by virtue of Lemma 1.2 , exist a constant $\mu_{1}$, such that $\xi=\mu_{1} w(x)$ has a common tangent with $\xi=v(x)$ at some point of this arch. Since $\dot{\mu} w(x)>v(x)$ in the interval in question, we would necessarily have $\mu_{1}<\mu$, and $\xi=\mu_{1} w(x)$ and $\xi=v(x)$ would intersect in the interval $\left(a_{n+2}, a_{n+3}\right)$. The solution $v(x)-\mu_{1} w(x)$ would thus have a double zero which is flanked by two other zeros (the one just mentioned and the double zero at $x=a$ ), and this contradicts Lemma 2.3. The number of interior zeros (all necessarily simple by Lemma 2.3) is thus equal to twice the number of positive arches of $\xi=v(x)$ in $\left(a, a_{n+2}\right)$ if $v(x)<0$ in $\left(a, a_{3}\right)$. If $v(x)>0$ in $\left(a, a_{3}\right)$, $\xi=v(x)$ and $\xi=\mu w(x)$ must have precisely one point of intersection in $\left(a, a_{3}\right)$. If this were not so, we would have $\mu w^{\prime \prime}(a)>v^{\prime \prime}(a)$ and a solution $v(x)-\mu_{2} w(x)$ $\left(\mu_{2}<\mu\right)$ would have a triple zero at $x=a$. As pointed out above, such a solution would also vanish in the interval $\left(a_{n+2}, a_{n+3}\right)$, and this contradicts Lemma 2.1. An elementary count shows therefore that in all cases the number of zeros of $w_{1}(x)$ in the closed interval $[a, \alpha]$ is precisely $n+3$. Since $\alpha<a_{n+3}$, this proves our assertion. To show the uniqueness of our solution we suppose that there exists another solution, say $w_{2}(x)$, such that $w_{2}(a)=w_{2}(\alpha)=0$ which likewise has $n+3$ zeros in $[a, \alpha]$. By Theorem 2.6, the zeros of $w_{2}(x)$ at $x=a$, $\alpha$ must be simple since otherwise $w_{1}(x)$ and $w_{2}(x)$ would be constant multiples of each other. By Corollary 3.2, the number of zeros of $w_{2}(x)$ in $(a, \alpha)$ may be at most larger by 1 than the number of zeros of $w_{1}(x)$ in this interval. But the zeros of $w_{1}(x)$ at $x=a, \alpha$ are double, while those of $w_{2}(x)$ are simple. The solution $w_{1}(x)$ has therefore more zeros in $[a, \alpha]$ than $w_{2}(x)$, and we have proved the following theorem.

Theorem 3.6. If there exists a solution $y(x)$ of (2.1) which vanishes for $x=a$ and has at least $n+3$ zeros in $[a, \infty)$, there then exist $n$ points $\eta_{1}, \cdots, \eta_{n}$ $\left(a<\eta_{1}<\eta_{2}<\cdots<\eta_{n}\right)$ and $n$ essentially unique solutions $y_{1}(x), \cdots, y_{n}(x)$ of (2.1) with the following properties:

(a) $y_{v}(x)$ has double zeros at $x=a$ and $x=\eta_{\nu}$;

(b) $y_{\nu}(x)$ has precisely $\nu+3$ zeros in $\left[a, \eta_{\nu}\right]$ (where the two double zeros are counted according to their multiplicities);

(c) any other solution $y(x)$ such that $y(a)=0$ has fewer than $\nu+3$ zeros in $\left[a, \eta_{\mathbf{v}}\right]$.

Properties (a) and (c) show that the point $\eta_{\nu}$ coincides with the $\nu$ th con- 
jugate point defined above. It is worth noting that, in the case of solutions $y(x)$ with a double zero at $x=a$, the argument leading to Theorem 3.6 yields the following more precise information about the location of the zeros of $y(x)$.

THEOREM 3.7. If $y(x)$ is a solution of (2.1) such that $y(a)=y^{\prime}(a)=0$, then the zeros of $y(x)$ in $(a, \infty)$ are separated by the points $\eta_{v}(a)$ conjugate to a (except when the last zero of $y(x)$ is double in which case it coincides with a conjugate point).

4. It is clear from the last two theorems that the conjugate points are closely associated with the oscillatory or nonoscillatory character of the equation. We shall now show that the following result holds.

THEOREM 3.8. Equation (2.1) is oscillatory if, and only if, for every positive value $x=a$ there exists an infinity of conjugate points $\eta_{\nu}(a)$.

According to the definition in $\S 1,(2.1)$ is oscillatory if, and only if, it has at least one oscillatory solution. If there exists such a solution $y(x)$, we may, for any given positive $a$ and for any positive integer $n$, determine a value $b(b>a)$ such that $y(x)$ has $n$ zeros in $(a, b)$. If $u(x)$ denotes the solution of (2.1) for which $u(a)=u^{\prime}(a)=u(b)=0, u(x)$ will thus-by Theorem 3.3certainly have at least $n$ zeros in $[a, b]$. Since $n$ is arbitrary, we may therefore conclude from Theorem 3.6 (or 3.7) that there exists an infinity of conjugate points which belong to $x=a$.

Conversely, if there are infinitely many conjugate points belonging to a given $x=a$, then it is shown by Theorem 3.6 that there exist solutions of (2.1) with arbitrarily many zeros in $(a, \infty)$; in fact, we may take the extremal solutions $y_{v}(x)$ defined in the statement of the theorem. This, however, is not enough; we have to show the existence of a solution with an infinity of zeros. Such a solution is easily produced by a limiting process. By the existence theorem for equation (1.1), all solutions of (2.1) which have a double zero at $x=a$ may be written in the form $y(x)=c_{1} u(x)+c_{2} v(x)$, where $c_{1}, c_{2}$ are constants and $u(x), v(x)$ are two such solutions which are not constant multiples of each other (we may, for instance, characterize $u(x)$ by the conditions $u^{\prime \prime \prime}(a)=0, u^{\text {iv }}(a)=1$ and $v(x)$ by $\left.v^{\prime \prime \prime}(a)=1, v^{\text {iv }}(a)=0\right)$. If, in addition, $y(x)$ is normalized by the condition $c_{1}^{2}+c_{2}^{2}=1$, the resulting class of solutions is trivially compact. If the solutions $y_{\nu}(x)$ of Theorem 3.6 are normalized in this manner, the sequence $\left\{y_{\nu}(x)\right\}$ will therefore possess a subsequence which converges-uniformly in any closed interval-to a solution $y_{0}(x)$ of (2.1). Any limit-point of zeros of the $y_{\nu}(x)$ belonging to the converging subsequence will be a zero of $y_{0}(x)$. By Theorem 3.7, all solutions $y_{\nu}(x)(\nu>n)$ vanish in the interval $\left(\eta_{n-1}, \eta_{n}\right)$. Hence, $y_{0}(x)$ must have a zero between any two consecutive conjugate points. This completes the proof of Theorem 3.8.

Theorem 3.8 enables us to establish the following basic result. 
THEOREM 3.9. If equation (2.1) is nonoscillatory, there then exists a positive a such that no solution of (2.1) has more than three zeros in $(a, \infty)$.

In the terminology introduced in $\$ 1$, we may also say that if $(2.1)$ is nonoscillatory, there exists a positive $a$ such that (2.1) is nonoscillatory in $(a, \infty)$. If this were not true, we could choose a sequence of points $x_{1}, x_{2}, \cdots$ $\left(0<x_{1}<x_{2}<\cdots\right)$ tending to infinity such that for each $x_{m}$ there exists a solution $y_{m}(x)$ of $(2.1)$ which has at least four zeros in $\left(x_{m}, \infty\right)$. We can, moreover, choose the $x_{m}$ in such a way that the point $x_{m+1}$ lies to the right of the fourth zero of $y_{m}(x)$ in $\left(x_{m}, \infty\right)$. If $v_{m}$ denotes the solution of (2.1) which has a double zero at $x=x_{1}$ and another zero at $x=x_{m}$ then, according to Theorem $3.5, v_{m}(x)$ must have a zero in each of the intervals $\left(x_{\nu}, x_{\nu+1}\right), \nu=1,2, \cdots$, $m-1$. Since $m$ may be any positive integer there will thus exist solutions of (2.1) with arbitrarily many zeros in $\left(x_{1}, \infty\right)$ and a double zero at $x=x_{1}$. Theorem 3.6 shows that this implies an infinity of conjugate points of $x=x_{1}$. By Theorem 3.8, (2.1) is therefore oscillatory and this contradicts our assumption.

5. We shall now show that the conjugate points of a given point $x=a$ are the zeros of a solution of a certain second-order differential equation associated with (2.1). To obtain this equation, we denote by $u(x)$ a solution of (2.1) which has a double zero at $x=a$ and is positive for $x>a$ (by Lemma 2.1 we may, for instance, take the solution $u(x)$ characterized by $u(a)=u^{\prime}(a)$ $\left.=u^{\prime \prime \prime}(a)=0, u^{\prime \prime}(a)=1\right)$, and we let $v(x)$ be any other solution of (2.1) with a double zero at $x=a$ which is not a constant multiple of $u(x)$. As observed above, all other solutions with a double zero at $x=a$ are of the form $w(x)$ $=A u(x)+B v(x)$, where $A$ and $B$ are constants. The conjugate points of $x=a$ coincide with those points at which some function $w(x)$ has a double zero. Since the points at which $A u(x)+B v(x)$ has a double zero are identical with the points at which the determinant

$$
\sigma(x)=v(x) u^{\prime}(x)-u(x) v^{\prime}(x)
$$

vanishes, the conjugate points are completely characterized by the condition $\sigma(x)=0$.

Both $u(x)$ and $v(x)$ are solutions of (2.1). Hence

$$
v\left(r u^{\prime \prime}\right)^{\prime \prime}-u\left(r v^{\prime \prime}\right)^{\prime \prime}=0 .
$$

Integrating this identity from $a$ to $x$ we obtain

$$
v(x)\left[r(x) u^{\prime \prime}(x)\right]^{\prime}-u(x)\left[r(x) v^{\prime \prime}(x)\right]^{\prime}-r(x)\left[v^{\prime}(x) u^{\prime \prime}(x)-u^{\prime}(x) v^{\prime \prime}(x)\right]=0 .
$$

Differentiating (3.1), we have $\sigma^{\prime}=v u^{\prime \prime}-u v^{\prime \prime}$,

$$
\left(r \sigma^{\prime}\right)^{\prime}=v\left(r u^{\prime \prime}\right)^{\prime}-u\left(r v^{\prime \prime}\right)^{\prime}+r\left(v^{\prime} u^{\prime \prime}-u^{\prime} v^{\prime \prime}\right),
$$

and thus, by (3.2), $\left(r \sigma^{\prime}\right)^{\prime}=2 r\left(v^{\prime} u^{\prime \prime}-u^{\prime} v^{\prime \prime}\right)$. Eliminating $v^{\prime}$ by means of (3.1), we arrive at the equation 


$$
\left(r \sigma^{\prime}\right)^{\prime}-2 r \frac{u^{\prime}}{u} \sigma^{\prime}+2 r \frac{u^{\prime \prime}}{u} \sigma=0,
$$

which must be satisfied by $\sigma(x)$. Since both $r$ and $u$ are positive for $x>a$, the differential equation (3.4) has no singularities in $(a, \infty)$. The point $x=a$ is, of course, a singularity of the equation, but the solution $\sigma(x)$ remains continuous at this point. As (3.3) shows, $\sigma(x)$ has a quadruple zero at $x=a$. It is easily confirmed that the point $x=a$ is a regular singular point of equation (3.4), and that one solution of (3.4) has a quadruple zero there. The other solution has a triple or simple zero at $x=a$, according as $u^{\prime \prime}(a)$ does or does not vanish. The function (3.1) is therefore completely characterized (except for a multiplicative constant) by being a solution of the differential equation (3.4) and having a quadruple zero at $x=a$.

The substitution $\sigma(x)=\tau(x) u(x)$ transforms (3.4) into

$$
\left(r \tau^{\prime}\right)^{\prime}+\left[\frac{\left(r u^{\prime}\right)^{\prime}}{u}+2 r\left(\frac{u^{\prime}}{u}\right)^{\prime}\right] \tau=0
$$

where $\tau(x)$ is now characterized by the condition $\tau(a)=0$. Since the zeros of $\tau(x)$ in $(a, \infty)$ coincide with the zeros of $\sigma(x)$ in this interval and the latter are identical with the points conjugate to $x=a$, we have established the following result.

THEOREM 3.10. If $u(x)$ is a solution of (2.1) which has a double zero at $x=a$ and is positive for $x>a$, the points $\eta_{\nu}(a)$ conjugate to $x=a$ coincide with the zeros of the solution $\tau(x)$ of (3.5) for which $\tau(a)=0$.

A comparison with Theorem 3.8 shows that the question whether or not (2.1) is oscillatory can be reduced to the corresponding question for the second-order equation (3.5), provided a solution $u(x)$ of $(2.1)$ with the requisite properties is known. As the following theorem shows, the resulting oscillation criterion can be further simplified by dropping the requirement that the solution $u(x)$ used in (3.5) have a double zero.

TheOREM 3.11. If $u(x)$ is a nonoscillatory solution of (2.1), then (2.1) will be oscillatory if, and only if, the second-order equation (3.5) is oscillatory.

Proof. As shown above, equation (3.5) is satisfied by $\sigma(x)=v(x) u^{\prime}(x)$ $-u(x) v^{\prime}(x)$, where $u(x)$ and $v(x)$ are two solutions of (2.1) which are related by the identity (3.2). We first suppose that (2.1) is nonoscillatory. By Theorem 3.9, there exists a positive value $a$ such that no solution of (2.1) has more than three zeros in $(a, \infty)$. There will further exist a number $x=b(b>a)$ such that $u(x) \neq 0$ for $x \geqq b$. Without losing generality, we may assume that $u(x)>0$ for $x \geqq b$. We now choose a solution $v(x)$ of (2.1) which, in addition to (3.2), satisfies the conditions $v(b)=v(c)$, where $c>b$. Since the solutions of (2.1) form a linear four-parameter family of functions, it is clear that such a solu- 
tion exists. If (3.5) is oscillatory, we can take $c$ large enough so that all solutions of (3.5) have at least three zeros in $(b, c)$. Since (2.1) is nonoscillatory in $(a, \infty)$ and $v(x)$ vanishes at $x=b$ and $x=c, v(x)$ cannot have more than one zero in $(b, c)$.

The solution $\sigma(x)=v(x) u^{\prime}(x)-u(x) v^{\prime}(x)$ of (3.4) has at least three zeros in $(b, c)$, and it follows therefore that there are at least two zeros of $\sigma(x)$ in $(b, c)$, say $x=x$, and $x=x_{2}$, at which $v(x)$ is of the same sign. Since $\sigma\left(x_{1}\right)$ $=\sigma\left(x_{2}\right)=0$, there exist constants $\alpha_{1}$ and $\alpha_{2}$ such that the solutions $w_{1}(x)$ $=u(x)+\alpha_{1} v(x)$ and $w_{2}(x)=u(x)+\alpha_{2} v(x)$ have double zeros at $x=x_{1}$ and $x=x_{2}$, respectively. In view of

$$
w_{1}(x)-w_{2}(x)=\left(\alpha_{1}-\alpha_{2}\right) v(x),
$$

we may therefore conclude that $w_{1}\left(x_{2}\right)$ and $w_{2}\left(x_{1}\right)$ are of opposite sign. Hence, one of these two numbers, say $w_{1}\left(x_{2}\right)$, must be negative. But this contradicts our assumption that $(2.1)$ is nonoscillatory in $(a, \infty)$. Indeed, since $v(b)$ $=v(c)=0$, both $w(b)$ and $w(c)$ are positive and $w_{1}(x)$ changes its sign an even number of times in $(b, c)$. Since $w_{1}(x)$ has a double zero at $x=x_{1}$ and is negative at $x=x_{2}, w_{1}(x)$ must therefore have at least four zeros in $(b, c)$. The assumption that (3.5) is oscillatory while (2.1) is not has thus led to a contradiction.

It remains to be shown that (3.5) is oscillatory if the same is true of (2.1). If $u(x)$ is a nonoscillatory solution of (2.1), we first show that there exist solutions $v(x)$ of (2.1) which are related by (3.2) to $u(x)$, and which have an arbitrary number of zeros in $(a, \infty)$. Such solutions are easily constructed by means of Theorem 3.1. Let $n$ be an arbitrary positive integer and let $v_{1}(x)$ be a solution of (2.1) which vanishes at $x=a, x=\eta_{n}(a)$, and at another point which does not coincide with a zero of $y_{n}(x)$ (cf. Theorem 3.6). Clearly, $v_{1}(x)$ is not a constant multiple of $y_{n}(x)$, and it follows from Theorem 3.1 that the zeros of $\lambda y_{n}(x)$ and $\mu v_{1}(x)$ in $\left(a, \eta_{n}(a)\right)$ separate each other, where $\lambda$ and $\mu$ are arbitrary constants. Since (by Lemma 2.3) all these zeros are simple, the curves $\xi=\lambda y_{n}(x)$ and $\xi=\mu v_{1}(x)$ must intersect in each interval between two consecutive zeros of $y_{n}(x)$. There are $n-2$ such intervals. Adding to this the common zero of $v_{1}(x)$ and $y_{n}(x)$ at $x=\eta_{n}(a)$, we thus find that, regardless of the values of $\lambda$ and $\mu$, the solution $\lambda y_{n}(x)-\mu v_{1}(x)$ has at least $n-1$ zeros in $(a, \infty)$.

If $S(u, v)$ denotes the expression

$$
S(u, v)=v\left(r u^{\prime \prime}\right)^{\prime}-u\left(r v^{\prime \prime}\right)^{\prime}-r\left(v^{\prime} u^{\prime \prime}-u^{\prime} v^{\prime \prime}\right),
$$

then, as shown above, $S(u, v)=$ const., if both $u(x)$ and $v(x)$ are solutions of (2.1). If $S\left(u, y_{n}\right)=c_{1}$ and $S\left(u, v_{1}\right)=c_{2}$, we obviously have $S\left(u, \lambda y_{n}-\mu v_{1}\right)$ $=\lambda c_{1}-\mu c_{2}$. If $\lambda$ and $\mu$ are suitably chosen, the solution $v(x)=\lambda y_{n}(x)-\mu v_{1}(x)$ will therefore satisfy the condition $S(u, v)=0$-which is identical with (3.2)and it will, moreover, have at least $n-1$ zeros in $(a, \infty)$. 
We now choose $a$ such that $u(x) \neq 0$ in $[a, \infty)$. Since $v(x)$ has at least $n-1$ zeros in $(a, \infty)$, it follows from Lemma 1.1 that $\sigma(x)=v(x) u^{\prime}(x)-u(x) v^{\prime}(x)$ has also at least $n-1$ zeros in $(a, \infty)$. Since $\sigma(x)$ is a solution of $(3.5)$ and $n$ is arbitrary, we conclude that (3.5) is oscillatory. This completes the proof of Theorem (3.11).

\section{The associated eigenvalue problem.}

1. In the mathematical treatment of the vibration of a rod extending from $x=a$ to $x=b$ and clamped at both ends a central role is played by the eigenvalue problem

$$
\left(r y^{\prime \prime}\right)^{\prime \prime}-\lambda p y=0, \quad y(a)=y^{\prime}(a)=y(b)=y^{\prime}(b)=0,
$$

where $r(x)$ and $p(x)$ are positive and belong to $C^{\prime \prime}$ and $C$, respectively, in $[a, b]$. The requirement that $y(x)$ have double zeros at $x=a, b$ may be replaced by other homogeneous boundary conditions - two at each end-in accordance with the physical conditions prescribed at the two end-points. We shall however be mainly concerned with the boundary conditions in (4.1) since the resulting eigenvalue problem is particularly helpful in the discussion of the oscillation properties of equation (2.1).

It is well known [3] that there exists a countable set of positive values $\lambda$ for which (4.1) has a solution $y(x)$ with the prescribed boundary values, and that the eigenvalues $\lambda$ have no finite limit-point. The lowest eigenvalue $\lambda_{1}$ may be characterized as the minimum of the Rayleigh quotient

$$
J(w)=\frac{\int_{a}^{b} r w^{\prime \prime 2} d x}{\int_{a}^{b} p w^{2} d x},
$$

where $w(x)$ ranges over the class of "admissible" functions which, in this case, are functions satisfying the same boundary conditions as $y(x)$ and having a piecewise continuous second derivative in $[a, b]$. If the $\lambda$ are arranged in an ascending sequence, then $\lambda_{n}$ may be defined by Courant's well-known minimax procedure [3] as $\lambda_{n}=\max \mu\left[g_{1}, \cdots, g_{n-1}\right]$ where $g_{1}(x), \cdots, g_{n-1}(x)$ may be any set of $n-1$ admissible functions and $\mu\left[g_{1}, \cdots, g_{n-1}\right]=\min J(w)$ where $w(x)$ ranges over the set of admissible functions for which

$$
\int_{a}^{b} p w g_{\nu} d x=0, \quad \quad \nu=1, \cdots, n-1 .
$$

We are concerned here only with those aspects of the eigenvalue problem (4.1) which can be utilized in the study of the oscillatory behavior of (2.1). Among the properties of (4.1) which fall under this heading there are two for which we did not succeed in finding proofs in the literature and which we accordingly prove here. 


\section{Lemma 4.1. All eigenvalues of problem (4.1) are simple.}

By this is meant that to each eigenvalue $\lambda$ there belongs exactly one eigenfunction $y(x)$ (except for a multiplicative constant). The proof of this result is very simple indeed. If there were two eigenfunctions say, $y_{1}(x)$ and $y_{2}(x)$, belonging to the same $\lambda$, then $y_{3}(x)=A y_{1}(x)+B y_{2}(x)$ would likewise be an eigenfunction of (4.1). The constants $A, B$ may be so chosen that $A^{2}+B^{2}>0$ and $y_{3}^{\prime \prime}(a)=0$. Since we have also $y_{3}(a)=y_{3}^{\prime}(a)=0$, it follows from Lemma 2.1 that $y_{3}(b) \neq 0$. But this contradicts the boundary conditions at $x=b$, and the lemma is'proved.

It may be of interest to point out that the same argument also proves the nondegeneracy of all positive eigenvalues in the more general problem in which the prescribed boundary conditions require two of the quantities $y, y^{\prime}$, $y^{\prime \prime},\left(r y^{\prime \prime}\right)^{\prime}$ to vanish at both ends of the interval (not necessarily the same two quantities in both cases). Indeed, if there exist two essentially different solutions associated with the same eigenvalue, then it is possible to form a linear combination for which three of these quantities vanish at $x=a$ and two at $x=b$, and this again contradicts Lemma 2.1. This argument fails, of course, if the problem has the eigenvalue $\lambda=0$, since Lemma 2.1 is valid only for equations (2.1) with positive $p(x)$. That the eigenvalue $\lambda=0$ may indeed be degenerate is shown by the equation (4.1) with the boundary conditions $y^{\prime \prime}(a)=y^{\prime \prime \prime}(a)=y^{\prime \prime}(b)=y^{\prime \prime \prime}(b)=0$, which has the eigenfunctions $y(x)=1$ and $y(x)=x$, both belonging to the eigenvalue $\lambda=0$. The statement occasionally found in the literature [e.g. 3; p. 297] -and illustrated by the preceding example - that the eigenvalues of the vibrating rod are in general not necessarily simple is thus found to apply (under the very general boundary conditions indicated) only to the lowest eigenvalue of the free-endpoint problem.

2. The other property of problem (4.1) which we require for our purposes is contained in the following statement.

Lemma 4.2. The nth eigenfunction $y_{n}(x)$ of the problem (4.1) has $n-1$ simple zeros in $(a, b)$.

A statement of this result may be found in Rayleigh's Theory of sound [vol. 1, p. 295], but Rayleigh apparently felt that a formal proof of this physically plausible fact was not required.

By Lemma 2.3, all the zeros of $y_{n}(x)$ in $(a, b)$ are simple. If $m$ denotes the number of these zeros, we show first that $m \leqq n-1$. To this end we remark that, by Theorem 3.6 , the point $x=b$ coincides with the conjugate point $\eta_{m+1}(a)$ of $x=a$. Accordingly, the equation

$$
\left(r y^{\prime \prime}\right)^{\prime \prime}-\lambda_{n} p y=0
$$

will also have solutions which satisfy the boundary conditions $y(a)=y^{\prime}(a)$ $=y\left(\eta_{\nu}\right)=y^{\prime}\left(\eta_{\nu}\right)=0$, where $\nu$ is any integer such that $1 \leqq \nu \leqq m$, and $\lambda_{n}$ will thus also play the role of an eigenvalue for the problem obtained from (4.1) 
by substituting $\eta_{v}$ for $b$. To estimate the order of the eigenvalue $\lambda_{n}$ of the problem associated with the interval $\left(a, \eta_{m}\right)$, we use the fact [3] that the $n$th eigenvalue of problem (4.1) is strictly decreasing if the fundamental interval increases. The $n$th eigenvalue of the problem related to $\left(a, \eta_{m}\right)$ is therefore larger than $\lambda_{n}$. By Lemma 4.1, this eigenvalue is simple and it follows that the order of the eigenvalue $\lambda_{n}$ of this problem is smaller than $n$. If we replace $\eta_{m}$ and $b$ by $\eta_{m-1}$ and $\eta_{m}$, respectively, the same argument shows that the order of the eigenvalue $\lambda_{n}$ of the problem associated with $\left(a, \eta_{m-1}\right)$ is smaller than $n-1$. Continuing in this fashion until we reach the first conjugate point, we find that the order of the eigenvalue $\lambda_{n}$ for this problem is smaller than $n-m+1$. Since this order cannot be smaller than 1 , it follows that $m \leqq n-1$.

To show that $m \geqq n-1$, we again use the monotonicity of the eigenvalues as functions of the fundamental interval. If, in problem (4.1), $b$ is replaced by a value $b^{\prime}$ such that $a<b^{\prime}<b$, an eigenvalue of given order will increase. Moreover, if $b^{\prime}$ varies continuously from $x=b$ to $x=a$, the $\nu$ th eigenvalue will increase continuously from $\lambda_{\nu}$ to $\infty$. Hence, if $\nu$ is an integer such that $1 \leqq \nu<n$, the $\nu$ th eigenvalue of (4.1) will increase continuously from $\lambda_{\nu}$ to $\infty$ if the upper limit of the interval varies from $x=b$ to $x=a$. Since by Lemma 4.1 , the eigenvalues are simple, we have $\lambda_{1}<\lambda_{2}<\cdots<\lambda_{n-1}<\lambda_{n}$, and there will therefore exist points $\eta_{\nu}(\nu=1, \cdots, n-1)$ in $(a, b)$ such that $\lambda_{n}$ is the $\nu$ th eigenvalue of the problem analogous to $(4.1)$ for the interval $\left(a, \eta_{\nu}\right)$. Lemma 4.1 shows further that these points are all distinct, and thus there are in $(a, b)$ at least $n-1$ conjugate points of $x=a$ with respect to equation (4.4). By Theorem 3.6, the number of zeros of the $n$th eigenfunction $y_{n}(x)$ of $(4.1)$ is therefore at least $n-1$. This completes the proof of Lemma 4.2.

3 . We close this section with the proof of a result for the differential equation

$$
y^{\text {iv }}-p(x) y=0, \quad p(x)>0 .
$$

THEOREM 4.3. If $n$ denotes the number of zeros of a solution $y(x)$ of equation (4.5) in the interval $[a, b]$, then

$$
n-3<\frac{1}{6} \int_{a}^{b}(x-a)^{3} p(x) d x .
$$

Proof. If $a_{1}$ is the first zero of $y(x)$ in $(a, b)$ then, by Theorem 3.6, the conjugate point $\eta_{n-3}$ of $x=a_{1}$ will be in $(a, b)$. It is therefore sufficient to prove our result for the case in which $y(x)$ has double zeros at $x=a, b$ and $x=b$ is the conjugate point of order $n-3$ of $x=a$. If $\lambda_{m}$ is the $m$ th eigenvalue of the problem

$$
y^{\text {iv }}-\lambda p(x) y=0, \quad y(a)=y^{\prime}(a)=y(b)=y^{\prime}(b)=0,
$$

it thus follows from Theorem (3.6) and Lemma (4.2) that we may set $\lambda_{n-3}=1$.

The problem (4.7) is equivalent to the integral equation 


$$
y(\xi)=\lambda \int_{a}^{b} G(x, \xi) p(x) y(x) d x
$$

where $G(x, \xi)$ is the Green's function of the differential operator $L(y) \equiv y^{\text {iv }}$ for the boundary conditions (4.7) [3]. If we set $u(x)=y(x)(p(x))^{1 / 2}$, the equation becomes $u(\xi)=\lambda \int_{a}^{b} K(x, \xi) u(x) d x$, where the kernel

$$
K(x, \xi)=G(x, \xi)(p(x) p(\xi))^{1 / 2}
$$

is symmetric in $x$ and $\xi$. Multiplying (4.7) by $y(x)$ and integrating from $a$ to $b$, we find that

$$
\int_{a}^{b} y^{\prime \prime 2} d x=\lambda \int_{a}^{b} p y^{2} d x
$$

which shows that all the eigenvalues are positive. The kernel (4.8) is therefore positive-definite, and we may apply Mercer's theorem [3]. This yields

$$
\sum_{\nu=1}^{\infty} \frac{1}{\lambda_{\nu}}=\int_{a}^{b} K(x, x) d x
$$

where $\lambda_{1}, \lambda_{2}, \cdots\left(0<\lambda_{1}<\lambda_{2}<\cdots\right)$ are the eigenvalues of the problem. Since $\lambda_{n-3}=1$, it follows that $\lambda_{\nu}<1$ for $1 \leqq \nu \leqq n-4$. Hence,

$$
n-3<\sum_{\nu=1}^{n-3} \frac{1}{\lambda_{\nu}}<\sum_{\nu=1}^{\infty} \frac{1}{\lambda_{\nu}}=\int_{a}^{b} K(x, x) d x=\int_{a}^{b} G(x, x) p(x) d x .
$$

In view of

$$
G(x, x)=\frac{(x-a)^{3}(b-x)^{3}}{6(b-a)^{3}} \leqq \frac{1}{6}(x-a)^{3}
$$

$[3 ; 6]$, this establishes $(4.6)$.

5. Comparison theorems.

1. In this section we are concerned with a comparison of the oscillatory behavior of solutions of the equation

$$
\left(r(x) y^{\prime \prime}\right)^{\prime \prime}-p(x) y=0, \quad r(x)>0, p(x)>0
$$

and

$$
\left(r_{1}(x) y^{\prime \prime}\right)^{\prime \prime}-p_{1}(x) y=0, \quad r_{1}(x)>0, p_{1}(x)>0,
$$

where the coefficients of the equations are related by the inequalities

$$
r(x) \geqq r_{1}(x), \quad p(x) \leqq p_{1}(x) .
$$


The basic result in this direction is given by the following theorem.

THEOREM 5.1. If $\eta_{n}(a)$ and $\eta_{n}^{\prime}(a)$ denote the nth conjugate points of $x=a$ for the equations (5.1) and (5.2), respectively, then

$$
\eta_{n}^{\prime}(a) \leqq \eta_{n}(a) \text {. }
$$

Proof. In view of our assumptions it follows from Theorem 3.6 and Lemma 4.2 that the $n$th eigenvalues of the problems

$$
\left(r y^{\prime \prime}\right)^{\prime \prime}-\lambda p y=0, \quad y(a)=y^{\prime}(a)=y\left(\eta_{n}\right)=y^{\prime}\left(\eta_{n}\right)=0
$$

and

$$
\left(r_{1} y^{\prime \prime}\right)^{\prime \prime}-\lambda^{\prime} p_{1} y=0, \quad y(a)=y^{\prime}(a)=y\left(\eta_{n}^{\prime}\right)=y^{\prime}\left(\eta_{n}^{\prime}\right)=0
$$

are both equal to 1 . We now compare problem (5.5) with the problem obtained from (5.6) by substituting $\eta_{n}$ for $\eta_{n}^{\prime}$, that is,

$$
\left(r_{1} y^{\prime \prime}\right)^{\prime \prime}-\lambda^{\prime \prime} p_{1} y=0, \quad y(a)=y^{\prime}(a)=y\left(\eta_{n}\right)=y^{\prime}\left(\eta_{n}\right)=0 .
$$

Since, for a given admissible function $w(x)$, the quotient (4.2) (with $b=\eta_{n}$ ) is not increased if $r$ and $p$ are replaced by $r_{1}$ and $p_{1}$, respectively, it follows from Courant's comparison theorem [3] that the $n$th eigenvalue of problem (5.7) is not larger than the $n$th eigenvalue of (5.5), that is, $\lambda_{n}^{\prime \prime} \leqq \lambda_{n}=1$. By an argument used in the preceding section, there will thus exist a uniquely determined point in $\left(a, \eta_{n}\right]$ such that the $n$th eigenvalue of problem (5.7) (with $x=\eta_{n}$ replaced by the point in question) is equal to 1 . But, as shown above, the $n$th eigenvalue of problem (5.6) is $\lambda_{n}^{\prime}=1$, and it follows that our point must coincide with $x=\eta_{n}^{\prime}$. Hence, $x=\eta_{n}^{\prime}$ lies in $\left(a, \eta_{n}\right]$, and our result is proved.

In view of the extremal property of the conjugate points (Theorem 3.6), Theorem 5.1 is, roughly speaking, equivalent to the statement that the fastest oscillation possible for a solution of (5.2) is at least as fast as the fastest oscillation permitted by equation (5.1). Comparison theorems for solutions other than those associated with the conjugate points may be obtained by combining Theorem 5.1 with the separation theorems of $\S 3$, in particular Theorems $3.1,3.3$ and 3.5. The following theorem is an example of the type of result obtainable in this way.

THEOREM 5.2. Let $u(x)$ and $v(x)$ be solutions of (5.1) and (5.2), respectively, where the coefficients of the two equations are related by (5.3). If $u(a)=v(a)$ $=u(b)=v(b)=0(a<b)$ and the number of zeros of $u(x)$ and $v(x)$ in $[a, b]$ is denoted by $n$ and $n^{\prime}(n \geqq 4)$, respectively, then $n^{\prime} \geqq n-1$.

Let $\eta_{\nu}(a)$ denote the $\nu$ th conjugate point of $x=a$ for equation (5.1). Then, by Theorem (3.6) and our assumptions, $a<\eta_{n-3}(a) \leqq b$. By Theorem 5.1, we thus have $a<\eta_{n-3}^{\prime}(a)<\eta_{n-3}(a) \leqq b$. If $v_{1}(x)$ denotes the nontrivial solution of (5.2) for which $v_{1}(a)=v_{1}^{\prime}(a)=v_{1}(b)=0$ it follows therefore from Theorem 3.7 
that the number of zeros of $v_{1}(x)$ in $[a, b]$ is at least $n$. By Corollary 3.2, the number of zeros of $v_{1}(x)$ in $(a, b)$ cannot exceed the number of zeros of $v(x)$ in this interval by more than 1 . However, if $v(x)$ has a simple zero at $x=a$ (and only this case has to be considered, since otherwise $v_{1}(x)$ and $v(x)$ are essentially identical), the extra zero of $v_{1}(x)$ implied by this statement cannot appear. This follows from Theorem 3.1 and the fact easily deduced from Lemma 2.3 and a slight modification of the argument leading to Lemma 1.2, that the first zero of $v(x)$ in $(a, b)$ precedes the first zero of $v_{1}(x)$ in this interval. The function $v(x)$ therefore has at least as many zeros in $(a, b)$ as $v_{1}(x)$. Because of the double zero of $v_{1}(x)$ at $x=a$ it follows therefore that $n^{\prime} \geqq n-1$, provided the zero of $v_{1}(x)$ at $x=b$ is simple. Since a double zero would imply that $x=b$ coincides with a conjugate point $\eta_{m}^{\prime}(a)(m>n-3)$, we would in this case even have $n^{\prime} \geqq n+1$. This completes the proof of Theorem 5.2.

2. We now turn to an entirely different type of comparison theorem. While the preceding results depended on the comparison of two fourth-order equations whose coefficients were related by (5.3), the following considerations will be based on the idea of comparing (2.1) with a second-order equation whose coefficients are related to those of $(2.1)$ in a suitable way. Our main result is the following.

THEOREM 5.3. Let $r(x)$ and $p(x)$ have the same meaning as in (2.1) and let $\sigma(x)$ be a positive function of class $C^{\prime}$ in $[a, b]$ such that the solution $u(x)$ of the differential equation

$$
\left(r(x) u^{\prime}\right)^{\prime}+\sigma(x) u=0
$$

which vanishes at $x=a$ does not vanish again in $(a, b)$. Let further $a_{1}, a_{2}, a_{3}, \cdots$ $\left(a<a_{1}<a_{2}<\cdots\right)$ denote the zeros in $(a, \infty)$ of the solution $v(x)$ of the differential equation

$$
(\sigma(x) v)^{\prime}+p(x) v=0
$$

which vanishes at $x=a$. If $\eta_{n}(a)$ denotes the nth conjugate point of $x=a$ for equation (2.1), then

$$
\eta_{n}(a) \geqq a_{n} .
$$

Proof. From our assumption regarding the solution $u(x)$ of $(5.8)$ it follows, by virtue of the Sturm comparison theorem, that there exists a constant $\alpha(\alpha \geqq 1)$ such that the equation

$$
(r w)^{\prime}+\alpha \sigma w=0
$$

has a solution which vanishes for $x=a, b$ and has no zeros in $(a, b)$. The number $\alpha$ is therefore the lowest eigenvalue of the problem $(r w)^{\prime}+\alpha \sigma w=0$, $w(a)=w(b)=0$. From the minimum property of the first eigenvalue we may thus conclude that the inequality 


$$
\alpha \int_{a}^{b} \sigma U^{2} d x \leqq \int_{a}^{b} r U^{\prime 2} d x
$$

holds for any function $U(x)$ of class $D^{\prime}$ in $[a, b]$ for which $U(a)=U(b)=0$.

We now use the minimax definition of the $n$th eigenvalue $\lambda_{n}$ of problem (4.1). As quoted in $\S 4, \lambda_{n}$ is equal to the minimum of the quotient (4.2), where $w(x)$ is a function of $D^{\prime \prime}$, has double zeros at $x=a, b$, and satisfies certain additional side conditions. If $w(x)$ is an admissible function of this type, then $w^{\prime}(x)$ clearly fulfills the conditions imposed on the functions $U(x)$ for which (5.11) holds. Because $\alpha \geqq 1$, we have

$$
J(w)=\frac{\int_{a}^{b} r w^{\prime \prime 2} d x}{\int_{a}^{b} p w^{2} d x} \geqq \alpha \frac{\int_{a}^{b} \sigma w^{\prime 2} d x}{\int_{a}^{b} p w^{2} d x} \geqq \frac{\int_{a}^{b} \sigma w^{\prime 2} d x}{\int_{a}^{b} p w^{2} d x}=J_{1}(w) .
$$

Now the functional $J_{1}(w)$ is the Rayleigh quotient which appears in the minimax definition of the eigenvalue $\mu_{n}$ of the problem

$$
\left(\sigma V^{\prime}\right)^{\prime}+\mu_{n} p V=0, \quad V(a)=V(b)=0 .
$$

The side conditions imposed on the admissible functions for this problem are based on the same orthogonality conditions (4.3) as the side conditions associated with the minimax problem leading to the eigenvalue $\lambda_{n}$ of (4.1). The boundary and admissibility conditions for the problem (4.1) are more stringent than those for (5.13): double zeros instead of simple zeros at the endpoints, and class $D^{\prime \prime}$ instead of class $D^{\prime}$. By Courant's comparison theorem, $\mu_{n}$ will therefore be not larger than the $n$th minimax value of $J_{1}(w)$. Since the $n$th minimax value of $J(x)$ for the same side conditions is $\lambda_{n}$, we may thus conclude from (5.12) that $\mu_{n} \leqq \lambda_{n}$.

If $b$ coincides with the conjugate point $\eta_{n}(a)$ then, according to Theorem 3.6 and Lemma $4.2, \lambda_{n}=1$. Hence, $\mu_{n} \leqq 1$, where $\mu_{n}$ is the $n$th eigenvalue of problem (5.13). By classical results the eigenfunction $V_{n}(x)$ of (5.13) has precisely $n+1$ zeros in $[a, b]$. Using the fact that $\mu_{n} \leqq 1$ and the Sturm comparison theorem we may therefore conclude that there exists a point $x=b^{\prime}$ in $(a, b]$ such that (5.9) has a solution $v(x)$ satisfying $v(a)=v\left(b^{\prime}\right)=0$ and vanishing $n-1$ times in $\left(a, b^{\prime}\right)$. Since $b^{\prime} \leqq b=\eta_{n}(a)$, the proof of Theorem 5.3 is complete.

3. Any special choice of the function $\sigma(x)$ which conforms to the conditions imposed on this function will give rise to an explicit comparison theorem. We shall illustrate the type of result obtainable in this way by two examples.

THEOREM 5.4. Let the coefficient $r(x)$ of (2.1) be such that 


$$
c=\frac{1}{\pi} \int_{a}^{\infty} \frac{d x}{r(x)}<\infty
$$

and let $v(x)$ be a solution of

$$
\left(r^{-1}(x) v^{\prime}\right)^{\prime}+c^{2} p(x) v=0
$$

for which $v(a)=0$. If $a_{1}, a_{2}, \cdots\left(a<a_{1}<a_{2}<\cdots\right)$ are the zeros of $v(x)$ in $(a, \infty)$ and $\eta_{n}(a)$ is the nth conjugate point of $x=a$ for equation (2.1), then

$$
\eta_{n}(a) \geqq a_{n} .
$$

This is the special case of Theorem 5.3 corresponding to $\sigma(x)=\left[c^{2} r(x)\right]^{-1}$. equation (5.8) reduces to

$$
c^{2}\left(r u^{\prime}\right)^{\prime}+\frac{u}{r}=0
$$

and this has the solution

$$
u=\sin \left[c^{-1} \int_{a}^{x} \frac{d x}{r(x)}\right]
$$

which vanishes for $x=a$ and is positive in $(a, \infty)$.

The next result refers to the equation

$$
y^{\mathrm{iv}}-p(x) y=0
$$

TheOREM 5.5. Let $w(x)$ be a solution of

$$
w^{\prime \prime}+\left[4 x^{2} p(x)-\frac{2}{x^{2}}\right] w=0
$$

for which $w(a)=0$ and let $a_{1}, a_{2}, \cdots\left(a<a_{1}<a_{2}<\cdots\right)$ be the zeros of $w(x)$ in $(a, \infty)$. If $\eta_{n}(a)$ is the nth conjugate point of $x=a$ for equation (5.14), then

$$
\eta_{n}(a) \geqq a_{n} .
$$

This is obtained by setting $\sigma(x)=(2 x)^{-2}$. Equation (5.8) then becomes $u^{\prime \prime}+(2 x)^{-2} u=0$, and this equation is nonoscillatory in $(0, \infty)$. Equation (5.9) reduces to

$$
\left(x^{-2} v^{\prime}\right)^{\prime}+4 p v=0 .
$$

If we set $v(x)=x w(x), w(x)$ satisfies equation (5.15), and the result follows.

Theorem 5.3 and its applications can easily be converted into statements regarding the maximum number of zeros which the solutions of (2.1) may have in a given interval. We confine ourselves to one example.

THEOREM 5.6. Let $y(x)$ be a solution of (5.14), and let $w(x)$ be a solution of the second-order equation (5.15). If $n$ and $m$ denote, respectively, the number of 
zeros which $y(x)$ and $w(x)$ have in a closed interval $[a, b](0<a<b<\infty)$, then $n \leqq m+3$.

Indeed, if $a^{\prime}$ denotes the first zero of $y(x)$ in $[a, b]$, the conjugate point $\eta_{n-3}\left(a^{\prime}\right)$ will, by Theorem 3.6, also be in $[a, b]$. By Theorem 5.5 , the solution of (5.15) which vanishes at $x=a^{\prime}$ will have at least $n-2$ zeros in $\left[a^{\prime}, \eta_{n-3}\left(a^{\prime}\right)\right]$. By the Sturm separation theorem, the number of zeros of this solution in $[a, b]$ will therefore be at least $n-3$, and the result follows. It may also be noted that the estimate may be improved to $n \leqq m+2$ if $w(x)$ is a solution of (5.14) which vanishes at one of the ends of the given interval.

6. Oscillation theorems.

1. In this section we derive a number of oscillation criteria for equation (2.1). There will be three types of such criteria. The first type is obtained directly from the comparison theorems of the preceding section. The second will be based on Theorem 6.6 of this section, which establishes a connection between the oscillation problem and the limiting case of a certain eigenvalue problem. The third class of oscillation criteria will be derived from Theorem 3.8 .

The criteria of the first type do not require further proof since they are immediate consequences of the comparison theorems of $\$ 5$ if the latter are combined with Theorem 3.8. Thus, Theorem 5.1 leads to the following result.

Theorem 6.1. If

$$
r(x) \geqq r_{1}(x)>0, \quad 0<p(x) \leqq p_{1}(x),
$$

and the equation

$$
\left(r_{1}(x) y^{\prime \prime}\right)^{\prime \prime}-p_{1}(x) y=0
$$

is nonoscillatory in $(a, \infty)$, then the same is true of the equation

$$
\left(r(x) y^{\prime \prime}\right)^{\prime \prime}-p(x) y=0 .
$$

It will be recalled from $\$ 1$ that equation (2.1) is said to be nonoscillatory in $(a, \infty)$ if no solution of $(2.1)$ has more than three zeros in $(a, \infty)$. In view of Theorem 3.6, the equation will therefore be nonoscillatory in $(a, \infty)$ if, and only if, there exists no conjugate point to $x=a$ in $(a, \infty)$.

As an example of the use of Theorem 6.1 we derive the following result.

THEOREM 6.2. If $\alpha$ is an arbitrary real constant, then equation (2.1) is oscillatory if

$$
\limsup _{x \rightarrow \infty} x^{-2-\alpha} r(x)<1, \quad \liminf _{x \rightarrow \infty} x^{2-\alpha} p(x)>\frac{\left(1-\alpha^{2}\right)^{2}}{16}
$$




$$
\liminf _{x \rightarrow \infty} x^{-2-\alpha} r(x)>1, \quad \limsup _{x \rightarrow \infty} x^{2-\alpha} p(x)<\frac{\left(1-\alpha^{2}\right)^{2}}{16} .
$$

The equation $y^{\mathrm{iv}}-p(x) y=0, p(x)>0$, is oscillatory if

$$
\liminf _{x \rightarrow \infty} x^{4} p(x)>\frac{9}{16},
$$

and nonoscillatory if

$$
\limsup _{x \rightarrow \infty} x^{4} p(x)<\frac{9}{16} .
$$

The result is obtained by using the Euler equation

$$
\left(x^{\alpha+2} y^{\prime \prime}\right)^{\prime \prime}-c^{2} x^{\alpha-2} y=0
$$

as an equation of comparison. Equation (6.1) has the solutions

$$
y=x^{(2 \mu+1-\alpha) / 2}
$$

where $\mu$ is any solution of

$$
\mu^{2}=\frac{1+\alpha^{2}}{4} \pm\left(\frac{\alpha^{2}}{4}+c^{2}\right)^{1 / 2}
$$

The equation is oscillatory if, and only if, $\mu^{2}<0$. Accordingly, (6.1) will change its oscillatory behavior if $c^{2}$ passes through the value $16^{-1}\left(1-\alpha^{2}\right)^{2}$. The second part of Theorem 6.1 corresponds to the special case $\alpha=-2$.

We next state the oscillation theorem which is implied by Theorem 5.3.

Theorem 6.3. Let $r(x)$ and $p(x)$ have the same meaning as in (2.1) and let $\sigma(x)$ be positive and of class $C^{\prime}$ in $(0, \infty)$. If the two second-order equations

$$
\left(r u^{\prime}\right)^{\prime}+\sigma u=0
$$

and

$$
\left(\sigma v^{\prime}\right)^{\prime}+p v=0
$$

are nonoscillatory in $(a, \infty)(0<a)$, then the same is true of equation (2.1).

Specific oscillation theorems are obtained by suitable specialization of $\sigma(x)$. For instance, the function $\sigma(x)=(2 x)^{-2}$ (used in obtaining Theorem 5.5 from Theorem 5.3) leads to the following result.

THEOREM 6.4. If

$$
y^{\prime \prime}+\left[4 x^{2} p(x)-\frac{2}{x^{2}}\right] y=0 \quad(p(x)>0)
$$

is nonoscillatory in $(a, \infty)$, the same is true of 


$$
y^{\mathrm{iv}}-p(x) y=0 .
$$

It may be observed that the last result is the best possible of its kind. Since the second-order equation $u^{\prime \prime}+q u=0$ is nonoscillatory for $q(x) \leqq(2 x)^{-2}$, it follows that (6.3) is nonoscillatory for $p(x) \leqq 9 x^{-4} / 16$. The constant $9 / 16$ cannot be improved upon since, as shown in connection with Theorem 6.2, the Euler equation $y^{\text {iv }}-(9 / 16+\epsilon) x^{-4} y=0$ is oscillatory for positive $\epsilon$.

Further nonoscillation criteria for (6.3) may be obtained by combining Theorem 6.4 with the known nonoscillation criteria for the second-order equation (6.2). For instance, the sufficient nonoscillation criterion

$$
\lim \sup _{x \rightarrow \infty} x \int_{x}^{\infty} q(x) d x<1 / 4
$$

for the equation $u^{\prime \prime}+q u=0$ [5] yields the following result.

Theorem 6.5. Equation (6.3) is nonoscillatory if

$$
\limsup _{x \rightarrow \infty} x \int_{x}^{\infty} x^{2} p(x) d x<\frac{9}{16} .
$$

This result is again sharp, as the above-mentioned Euler equation shows. If the nonoscillation criterion $\int^{\infty} x q(x) d x<\infty$ [5] is applied to (6.2), equation (6.3) is found to be nonoscillatory provided

$$
\limsup _{x \rightarrow \infty} \frac{1}{\log x} \int_{a}^{\infty} x^{3} p(x) d x<\frac{1}{2} .
$$

The constant $1 / 2$, is however, not sharp (the true constant is $9 / 16$, as may be shown by a suitable strengthening of the second-order criterion used). It may be noted that the weaker criterion $\int_{a}^{\infty} x^{3} p(x) d x<\infty$ can also be obtained as a direct consequence of Theorem 4.3.

2. A different type of oscillation theorem may be obtained by means of the following result which establishes a connection between the oscillation problem and a limiting case of the eigenvalue problem (4.1).

THEOREM 6.6. Equation (2.1) is nonoscillatory in $(a, \infty)$ if, and only if, the lowest eigenvalue $\lambda=\lambda(b)$ of the problem

$$
\left(r y^{\prime \prime}\right)^{\prime \prime}-\lambda p y=0, \quad y(a)=y^{\prime}(a)=y(b)=y^{\prime}(b)=0
$$

satisfies $\lambda(b)>1$ for all $b>a$.

As mentioned above, equation (2.1) is nonoscillatory in $(a, \infty)$ if, and only if, the point $x=a$ has no conjugate points in $(a, \infty)$. Hence, if $(2.1)$ is oscillatory in $(a, \infty)$, there will exist a first conjugate point $\eta_{1}(a)$ in this interval. In view of Theorem 3.6 and Lemma 4.2 , it follows that $\lambda(b)=1$ for $b=\eta_{1}(a)$. Because of the monotonicity of $\lambda(b)$, we have, moreover, $\lambda(b)<1$ 
for $b>\eta_{1}(a)$. The condition $\lambda(b)>1$ is thus found to be sufficient for nonoscillation in $(a, \infty)$.

To show the condition is necessary we suppose there exists a number $b>a$ such that $\lambda(b)<1$. Since $\lambda(b)$ increases monotonically and continuously if $b$ decreases, and tends to $\infty$ for $b \rightarrow a$, there exists a value $b^{\prime}$ in $(a, b)$ such that $\lambda\left(b^{\prime}\right)=1$. By Theorem 3.6 and Lemma $4.2, b^{\prime}$ coincides with the first conjugate point $\eta_{1}(a)$, and the existence of such a point is incompatibie with nonoscillation in $(a, \infty)$. This proves the necessity.

Since $\lambda(b)$ is the minimum of the Rayleigh quotient (4.2) as $w(x)$ ranges over the class of all functions of $D^{\prime \prime}(a, b)$ which have double zeros at $x=a, b$, Theorem 6.6 leads to the following very general nonoscillation criterion.

THEOREM 6.7. Let $b$ be such that $b>a$, and let $w(x)$ be a function of class $D^{\prime \prime}$ in $(a, b)$ such that $w(a)=w^{\prime}(a)=w(b)=w^{\prime}(b)=0$. If equation (2.1) is nonoscillatory in $(a, \infty)$, then

$$
\int_{a}^{b} p w^{2} d x \leqq \int_{a}^{b} r w^{\prime \prime 2} d x
$$

Every function $w(x)$ with the required properties will thus give rise to a necessary criterion for nonoscillation. We confine ourselves here to a few examples which will illustrate the possibilities inherent in this procedure.

We choose a positive number $h$ such that $a<a+h<c-h<c<c+h<2 c$ $+a-h<2 c+a=b$, and we define a function $w(x)$ as follows:

$$
\begin{aligned}
w(x) & =(x-a)^{2}, & & x \in[a, a+h] \\
w(x) & =h(2 x-2 a-h), & & x \in[a+h, c-h] \\
w(x) & =2 h(c-a-h)-(x-c)^{2}, & & x \in[c-h, c+h] \\
w(x) & =h(4 c-2 x-h-2 a), & & x \in[c+h, 2 c+a-h] \\
w(x) & =(2 c+a-x)^{2}, & & x \in[2 c+a-h, 2 c+a] .
\end{aligned}
$$

Both $w(x)$ and $w^{\prime}(x)$ are continuous in $(a, 2 c+a)$, while $w^{\prime \prime}(x)$ is continuous except for four finite jumps. Since $w(x)$ has, moreover, double zeros at $x=a, 2 c$, it may be used in (6.4). If, on the left-hand side of (6.4), we integrate only over the interval $(a+h, c-h)$ (which is permissible since the integrand is positive) we obtain

$$
h^{2} \int_{a+h}^{c-h}\left(x-a-\frac{h}{2}\right)^{2} p(x) d x \leqq \int_{a}^{a+h} r d x+\int_{c-h}^{c+h} r d x+\int_{2 c-h+a}^{2 c+a} r d x .
$$

If $r(x) \leqq m$ in $(a, \infty)$, we thus have

$$
h^{2} \int_{a+h}^{c-h}\left(x-a-\frac{h}{2}\right)^{2} p(x) d x \leqq 4 m h .
$$


Since the right-hand side is independent of $c$, it follows that the integral

$$
\int_{a}^{\infty}\left(x-a-\frac{h}{2}\right)^{2} p(x) d x
$$

-and, therefore, also the integral

$$
\int_{a}^{\infty} x^{2} p(x) d x
$$

- exists, and that

$$
\int_{a+h}^{\infty}\left(x-a-\frac{h}{2}\right)^{2} p(x) d x \leqq \frac{4 m}{h}
$$

If we set $x_{0}=a+h$ and observe that $x-a-h / 2>x-x_{0}$, we have proved the following theorem.

Theorem 6.8. If $r(x) \leqq m$ and (2.1) is nonoscillatory in $(a, \infty)$, then

$$
\int_{a}^{\infty} x^{2} p(x) d x<\infty
$$

and

$$
\int_{x_{0}}^{\infty}\left(x-x_{0}\right)^{2} p(x) d x \leqq \frac{4 m}{x_{0}-a}, \quad a<x_{0} .
$$

A more general oscillation theorem is obtained if the function $w(x)$ of Theorem 6.7 is defined as follows.

$$
\begin{array}{lll}
w(x)=(x-a)^{2}, & x \in[a, a+h] \\
w(x)=h^{2}\left[1+\frac{2}{h \nu}(x-a-h)\right]^{\nu}, & x \in[a+h, c-h] \\
w(x)=A-B(x-c)^{2}, & x \in[c-h, c+h] \\
w(x)=A-B h^{2}-2 B h(x-c-h), & x \in[c+h, b-h] \\
w(x)=B(x-b)^{2}, & x \in[b-h, b]
\end{array}
$$

where $\nu \neq 0$,

$$
\begin{aligned}
& a<a+h<c-h<c+h<b-h<b, \\
& A=\frac{2 h}{\nu}[c-a-(2-\nu) h]\left[1+\frac{2}{h \nu}(c-a-2 h)\right]^{\nu-1}, \\
& B=\left[1+\frac{2}{h \nu}(c-a-2 h)\right]^{\nu-1},
\end{aligned}
$$


and

$$
b=c+h+\frac{A}{2 B h} .
$$

It is easily verified that $v(x)$ is an admissible function; it may therefore be used in (6.4). Since $w^{\prime \prime}(x)=0$ in $(c+h, b-h)$, the righthand side of (6.4) reduces to

$$
\frac{16(\nu-1)^{2}}{\nu^{2}} \int_{a+h}^{c-h}\left[1+\frac{2}{h \nu}(x-a-h)\right]^{2 \nu-4} r(x) d x
$$

and the sum of the three integrals

$$
4 \int_{a}^{a+h} r(x) d x, \quad 4 B^{2} \int_{c-h}^{c+h} r(x) d x, \quad 4 B^{2} \int_{b-h}^{b} r(x) d x .
$$

If $r(x) \leqq m$ and $h$ is fixed, this sum is bounded by $4 m h\left(1+3 B^{2}\right)$, and this bound is independent of $c$. If the integral

$$
\int_{a}^{\infty} x^{2 \nu-4} r(x) d x
$$

exists, the expression (6.5) will also be bounded, and the same will be true of the left-hand side of (6.4). If we utilize only the integral over the subinterval $(a+h, c-h)$, we thus obtain the boundedness of

$$
h^{4} \int_{a+h}^{c-h}\left[1+\frac{2}{h \nu}(x-a-h)\right]^{2 \nu} p(x) d x
$$

for arbitrarily large $c$. But this is equivalent to the existence of the integral

$$
\int_{a}^{\infty} x^{2 v} p(x) d x
$$

and we have the following result.

THEOREM 6.9. Let $\mu$ be a positive constant and let (2.1) be nonoscillatory in $(a, \infty)$. If $r(x)$ is such that $r(x)<m<\infty$ and

$$
\int_{a}^{\infty} x^{\mu-4} r(x) d x<\infty,
$$

then $p(x)$ must satisfy the condition

$$
\int_{a}^{\infty} x^{\mu} p(x) d x<\infty .
$$

If $r(x)=1,(6.6)$ holds for any $\mu$ such that $\mu<3$. In this case, Theorem 6.9 will accordingly take the following form. 
Corollary 6.10. If

$$
y^{\mathrm{iv}}-p(x) y=0
$$$$
p(x)>0
$$

is nonoscillatory in $(a, \infty)$, then

$$
\int_{a}^{\infty} x^{\mu} p(x) d x<\infty
$$

for any $\mu<3$.

As the nonoscillatory equation $y^{\mathrm{iv}}-(9 / 16) x^{-4} y=0$ shows, the theorem does not hold for $\mu=3$. The bound given is therefore the best of its kind. It is also worth noting that, as shown above, the inequality (6.8), with $\mu=3$, is a sufficient condition for the nonoscillation of (6.7).

3. We finally show how Theorem 3.8 may be used in order to obtain sufficient criteria for nonoscillation. If $(2.1)$ is oscillatory and $x=a$ is such that $a>0$, then, according to Theorem 3.8 , there always exists a point $b(b>a)$ and a nontrivial solution $y(x)$ of $(2.1)$ such that $y(a)=y^{\prime}(a)=y(b)=y^{\prime}(b)=0$. Multiplying (2.1) by $y(x)$ and integrating from $a$ to $b$, we have therefore

$$
\int_{a}^{b} p y^{2} d x=\int_{a}^{b} r y^{\prime \prime 2} d x
$$

On the other hand,

$$
y(x)=\int_{a}^{x}(x-t) y^{\prime \prime}(t) d t
$$

thus,

$$
y^{2}(x) \leqq \int_{a}^{x} \frac{(x-t)^{2}}{r(t)} d \iota \int_{a}^{x} r(t) y^{\prime \prime 2}(t) d t
$$

and, if $a<x<b$,

$$
y^{2}(x) \leqq \int_{a}^{x} \frac{(x-t)^{2}}{r(t)} d t \int_{a}^{b} r(t) y^{\prime \prime 2}(t) d t .
$$

If we multiply both sides by $p(x)$, integrate from $a$ to $b$, and observe (6.9), we arrive at the inequality

$$
1 \leqq \int_{a}^{b} p(x)\left[\int_{a}^{x} \frac{(x-t)^{2}}{r(t)} d t\right] d x .
$$

Since

$$
\int_{a}^{x} \frac{(x-t)^{2}}{r(t)} d t \leqq(x-a)^{2} \int_{a}^{x} \frac{d t}{r(t)} \leqq(x-a)^{2} \int_{a}^{b} \frac{d t}{r(t)},
$$


it follows from (6.10) that

$$
1 \leqq \int_{a}^{b}(x-a)^{2} p(x) d x \int_{a}^{b} \frac{d x}{r(x)}
$$

and, if $r(x)$ is such that

$$
\int_{a}^{\infty} \frac{d x}{r(x)}=M<\infty
$$

then

$$
1 \leqq M \int_{a}^{b}(x-a)^{2} p(x) d x \leqq M \int_{a}^{b} x^{2} p(x) d x .
$$

If (2.1) is oscillatory then, again by Theorem 3.8 , there exists a conjugate point $b^{\prime}$ of $b\left(b^{\prime}>b\right)$, and the procedure just used will lead us to the inequality

$$
1 \leqq M \int_{b}^{b^{\prime}} x^{2} p(x) d x
$$

Continuing in this fashion and adding the inequalities obtained, we find that for any given positive integer $n$ there exists a positive number $b(n)$ such that

$$
n \leqq M \int_{a}^{b(n)} x^{2} p(x) d x
$$

This implies the following result.

THEOREM 6.11. If the integrals

$$
\int_{a}^{\infty} \frac{d x}{r(x)}, \quad \int_{a}^{\infty} x^{2} p(x) d x
$$

exist, then (2.1) is nonoscillatory (cf. [7, p. 45] and [8, p. 135]).

A more sensitive criterion is obtained by introducing the quantity

$$
\sigma(x)=\int_{x}^{\infty}(t-x)^{2} p(t) d t
$$

where $p(x)$ is such that the integral exists. Since

$$
\sigma^{\prime}(x)=-2 \int_{x}^{\infty}(t-x) p(t) d t, \sigma^{\prime \prime}(x)=2 \int_{x}^{\infty} p(t) d t, \sigma^{\prime \prime \prime}(x)=-2 p(x),
$$

(6.10) may be written

$$
1 \leqq-\frac{1}{2} \int_{a}^{b} \sigma^{\prime \prime \prime}(x)\left[\int_{a}^{x} \frac{(x-t)^{2}}{r(t)} d t\right] d x
$$


Integrating by parts three times and observing (6.12), we have

$$
\begin{aligned}
1 & \leqq-\frac{1}{2}\left[\sigma^{\prime \prime}(x) \int_{a}^{x} \frac{(x-t)^{2}}{r(t)} d t\right]_{a}^{b}+\int_{a}^{b} \sigma^{\prime \prime}(x)\left[\int_{a}^{x} \frac{(x-t)}{r(t)} d t\right] d x \\
& \leqq \int_{a}^{b} \sigma^{\prime \prime}(x)\left[\int_{a}^{x} \frac{(x-t)}{r(t)} d t\right] d x \\
& =\left[\sigma^{\prime}(x) \int_{a}^{x} \frac{(x-t)}{r(t)} d t\right]_{a}^{b}-\int_{a}^{b} \sigma^{\prime}(x)\left[\int_{a}^{x} \frac{d t}{r(t)}\right] d x \\
& \leqq-\int_{a}^{b} \sigma^{\prime}(x)\left[\int_{a}^{x} \frac{d t}{r(t)}\right] d x \\
& =-\left[\sigma(x) \int_{a}^{x} \frac{d t}{r(t)}\right]+\int_{a}^{b} \frac{\sigma(x)}{r(x)} d x \\
& \leqq \int_{a}^{b} \frac{\sigma(x)}{r(x)} d x .
\end{aligned}
$$

If we repeat this procedure for successive conjugate points, we are led to the following result.

Theorem 6.12. Let $p(x)$ be such that

$$
\tau(x)=\int_{x}^{\infty} t^{2} p(t) d t<\infty .
$$

If

$$
\int_{a}^{\infty} \frac{\tau(x)}{r(x)} d x<\infty
$$

then (2.1) is nonoscillatory.

The function $\tau(x)$ has been substituted for $\sigma(x)$ for the sake of simpler formulation. It is obvious that this does not affect the result.

7. Growth of solutions in the nonoscillatory case. In this section we shall show that the asymptotic growth of a solution $u(x)$ of a nonoscillatory equation

$$
u^{\mathrm{iv}}-p(x) u=0,
$$$$
p(x)>0,
$$

is subject to rather sharp restrictions. A similar treatment is possible in the case of the general equation (2.1), but the required computations become very involved. We prove the following theorem.

THEOREM 7.1. If equation (7.1) is nonoscillatory and $u(x)$ is a solution of (7.1), then for sufficiently large $x$, 


$$
A x^{-1 / 3} \leqq|u(x)| \leqq B x^{10 / 3},
$$

where $A$ and $B$ are positive constants.

The proof will be based on the properties of the second-order equation (3.5). According to Theorem 3.11, this equation will be oscillatory if and only if equation (2.1) is oscillatory. We are here concerned with the special case $r(x)=1$, and (3.5) reduces therefore to

$$
\tau^{\prime \prime}+\left[3\left(\frac{u^{\prime}}{u}\right)^{\prime}+\left(\frac{u^{\prime}}{u}\right)^{2}\right] \tau=0
$$

where $u(x)$ is any nonoscillatory solution of (7.1). Theorem 7.1 will be proved if we can show that any function $u(x)$, which does not vanish in $(a, \infty)$ and is such that equation (7.3) is nonoscillatory, is subject to the restrictions stated.

This will be accomplished by means of the following necessary nonoscillation conditions for second-order equations [10]: If $\tau^{\prime \prime}+q(x) \tau=0(q(x)>0)$ is nonoscillatory in $(a, \infty)$, then

$$
(x-a)^{1-\beta} \int_{a}^{x}(t-a)^{\beta} q(t) d t \leqq \frac{\beta^{2}}{4(\beta-1)},
$$

and

$$
(x-a) \int_{x}^{\infty} q(t) d t \leqq 1 .
$$

However, before we can apply these criteria, we have to show that it is sufficient to consider cases in which the coefficient of $\tau$ in (7.3) is positive for large values of $x$. This coefficient will be positive if

$$
\phi(x)=3 u(x) u^{\prime \prime}(x)-2 u^{\prime 2}(x)
$$

is positive. If $\phi(x)$ changes its sign an infinite number of times, the same will be true of $\phi^{\prime \prime}(x)$. Since a computation (using (7.1)) shows that

$$
\phi^{\prime \prime}(x)-\phi^{\prime \prime}(c)=3 p(x) u^{2}(x)-3 p(c) u^{2}(c)+2 \int_{c}^{x} p(t) u(t) u^{\prime}(t) d t
$$

we shall in this case have the relation

$$
3 p(c) u^{2}(c)=3 p\left(x_{v}\right) u^{2}\left(x_{v}\right)+2 \int_{c}^{x_{v}} p(t) u(t) u^{\prime}(t) d t
$$

for an infinite sequence of points $x=x_{\nu}$ such that $x_{\nu} \rightarrow \infty$ for $\nu \rightarrow \infty$, and a suitable point $x=c$.

In the proof of the left-hand inequality (7.2) it is evidently sufficient to 
consider solutions $u(x)$ such that $u(x) \rightarrow 0$ for $x \rightarrow \infty$. Since $u(x)$ and $u^{\prime}(x)$ are ultimately of constant sign, we also have $u^{\prime}(x) \rightarrow 0$ for $x \rightarrow \infty$, and $u(x) u^{\prime}(x)$ must be negative for large $x$. It follows from (7.6) that for such solutions $u(x)$ we have

$$
3 p(c) u^{2}(c)<3 p\left(x_{\nu}\right) u^{2}\left(x_{\nu}\right),
$$

provided $c$ has been taken large enough in the first place. Since equation (7.1) is nonoscillatory, we may conclude from Corollary 6.10 that $p(x) \rightarrow 0$ for $x \rightarrow \infty$. The same is true of $u(x)$, and it thus follows that $u(c)=0$. But $x=c$ may be identified with any of the sufficiently large zeros of $\phi^{\prime \prime}(x)$, and this conflicts with the assumption that (7.1) is nonoscillatory.

In the proof of the left-hand inequality (7.2), we may therefore restrict ourselves to functions $u(x)$ for which $\phi(x)$ is ultimately of constant sign.

To show that the same assumption may be made in the proof of the righthand inequality (7.2), we write (7.1) in the equivalent form

$$
u(x)=\sum_{\nu=0}^{3} \frac{u^{(\nu)}(a)}{\nu !}(x-a)^{\nu}+\frac{1}{6} \int_{a}^{x}(x-t)^{3} p(t) u(t) d t .
$$

This shows that

$$
u(x) \leqq x^{3}\left(C_{1}+C_{2} \int_{a}^{x} p(t) u(t) d t\right)
$$

where $C_{1}$ and $C_{2}$ are suitable positive constants. Since the exponent appearing in the right-hand inequality (7.2) is larger than 3 , we may therefore confine ourselves to the case in which $p(x) u(x)$ is not summable in $(a, \infty)$, as otherwise the right-hand inequality (7.2) would be trivially true. For the same reason we may also assume that both $u^{\prime}(x)$ and $u^{\prime \prime}(x)$ are ultimately positive. If $c$ is taken large enough, we may thus conclude from (7.6) that

$$
3 p(c) u^{2}(c) \geqq 2 u^{\prime}(c) \int_{c}^{x_{\nu}} p(t) u(t) d t .
$$

But this is absurd since, as just shown, the integral diverges if $x_{\nu} \rightarrow \infty$. Hence, the quantity $\phi(x)$ may in all cases be assumed to be ultimately of constant sign.

In order to show that we must ultimately have $\phi(x)>0$, we observe that $9\left(u^{1 / 3}\right)^{\prime \prime}=u^{-5 / 3} \phi(x)$. If $\phi(x)$ is negative, $u^{1 / 3}$ will therefore be concave. If $u(x)$ is positive and increasing, it follows that $u^{1 / 3}(x) \leqq A+B x$, where $A$ and $B$ are suitable constants. In this case $u(x)$ will therefore be $O\left(x^{3}\right)$, and the righthand inequality (7.2) holds trivially. If $u(x)$ is decreasing, $u^{1 / 3}(x)$ cannot be concave without ultimately becoming negative. It thus follows that in all cases of interest we may assume that $\phi(x)$-and therefore also the coefficient of $\tau$ in (7.3)-is ultimately positive.

We now take $a$ sufficiently large so that both $u(x)$ and the coefficient 
of $\tau$ in (7.3) are positive in $[a, \infty)$, and we apply the criterion (7.4). We obtain

$$
(x-a)^{1-\beta} \int_{a}^{x}(t-a)^{\beta}\left[3\left(\frac{u^{\prime}}{u}\right)^{\prime}+\left(\frac{u^{\prime}}{u}\right)^{2}\right] d t \leqq \frac{\beta^{2}}{4(\beta-1)}, \quad \beta>1 .
$$

Integrating by parts, we have

$$
\begin{aligned}
3(x-a) \frac{u^{\prime}(x)}{u(x)}-3 \beta(x-a)^{1-\beta} & \int_{a}^{x}(t-a)^{\beta-1} \frac{u^{\prime}}{u} d t \\
& +(x-a)^{1-\beta} \int_{a}^{x}(t-a)^{\beta}\left(\frac{u^{\prime}}{u}\right)^{2} d t \leqq \frac{\beta^{2}}{4(\beta-1)} .
\end{aligned}
$$

With the abbreviation

$$
\sigma(x)=(x-a) \frac{u^{\prime}(x)}{u(x)}
$$

this may be written

$$
\begin{aligned}
& 3 \sigma(x)-3 \beta(x-a)^{1-\beta} \int_{a}^{x}(t-a)^{\beta-2} \sigma(t) d t \\
& \quad+(x-a)^{1-\beta} \int_{a}^{x}(t-a)^{\beta-2} \sigma^{2}(t) d t \leqq \frac{\beta^{2}}{4(\beta-1)} .
\end{aligned}
$$

We first assume that $u^{\prime}(x)>0$, and we consider the positive quantity $\psi(x)$ defined by

$$
\psi^{2}(x)=(\beta-1)(x-a)^{1-\beta} \int_{a}^{x}(t-a)^{\beta-2} \sigma^{2}(t) d t .
$$

An application of the Schwarz inequality shows that

$$
(x-a)^{1-\beta} \int_{a}^{x}(t-a)^{\beta-2} \sigma(t) d t \leqq(\beta-1)^{-1} \psi(x) .
$$

We may therefore conclude from (7.8) and (7.9) that

$$
3(\beta-1) \sigma(x)-3 \beta \psi(x)+\psi^{2}(x) \leqq \beta^{2} / 4,
$$

that is,

$$
\left(\psi(x)-\frac{3}{2} \beta\right)^{2}+3(\beta-1) \sigma(x) \leqq \frac{5}{2} \beta^{2} .
$$

This inequality holds for any $\beta$ such that $\beta>1$. When $\beta=2$, we find that $\sigma(x) \leqq 10 / 3$. If we choose a value $b$ such that $a<b<x$, and observe the definition (7.7) of $\sigma(x)$, we obtain 


$$
u(x) \leqq u(b)(b-a)^{-10 / 3}(x-a)^{10 / 3}
$$

and this establishes the right-hand inequality (7.2).

To obtain a lower bound for $u(x)$ we employ the criterion (7.5). This leads to

$$
(x-a) \int_{x}^{T}\left[3\left(\frac{u^{\prime}}{u}\right)^{\prime}+\left(\frac{u^{\prime}}{u}\right)^{2}\right] d t \leqq 1,
$$

where the use of a finite upper integration limit is permissible since the integrand is positive. Integrating we obtain

$$
3 \frac{u^{\prime}(T)}{u(T)}-3 \frac{u^{\prime}(x)}{u(x)}+\int_{x}^{T}\left(\frac{u^{\prime}}{u}\right)^{2} d t \leqq \frac{1}{x-a} .
$$

Hence,

$$
(x-a) \frac{u^{\prime}(x)}{u(x)} \geqq-\frac{1}{3}+(x-a) \frac{u^{\prime}(T)}{u(T)},
$$

and the left-hand inequality (7.2) follows provided it can be shown that $u^{\prime}(T) / u(T) \rightarrow 0$ for $T \rightarrow \infty$.

Since $u^{\prime}(t)$ is negative in $(x, \infty)$, the quantity

$$
R(t)=-\frac{u^{\prime}(t)}{u(t)}
$$

is positive. It follows from (7.10) that

$$
\int_{x}^{T} R^{2}(t) d t \leqq 3 R(T)-3 R(x)+\frac{1}{x-a} \leqq 3 R(T)+C,
$$

where $C$ is independent of $T$. If we define a function $\xi(T)$ by

$$
\xi(T)=\int_{x}^{T} R^{2}(t) d t
$$

this takes the form

$$
\xi(T) \leqq 3\left(\xi^{\prime}(T)\right)^{1 / 2}+C .
$$

If $\xi(T)>C$, it follows that

$$
\frac{9 \xi^{\prime}(T)}{[\xi(T)-C]^{2}} \geqq 1
$$

Integrating we have

$$
\frac{9}{\xi(x)-C}-\frac{9}{\xi(T)-C} \geqq(T-x),
$$


whence

$$
\frac{9}{\xi(T)-C} \leqq \frac{9}{\xi(x)-C}-(T-x) .
$$

If $T$ is taken sufficiently large, this contradicts the assumption $\xi(T)>C$. If $\xi(T) \leqq C$ for all $T$, the integral (7.11) wiil converge as $T \rightarrow \infty$, and it follows that $R(T) \rightarrow 0$ as $T$ tends to infinity. This completes the proof.

It may be noted that the nonoscillatory equation $y^{\text {iv }}-9(2 x)^{-4} y=0$ has the solutions $x^{\nu}$, with $\nu=2^{-1}\left(3 \pm 10^{1 / 2}\right)$. This is rather close to the bounds given in (7.2).

\section{PART II}

8. General properties of solutions of (1.5).

1. We now pass to the discussion of the equation

$$
\left(r(x) y^{\prime \prime}\right)^{\prime \prime}+p(x) y=0, \quad r(x)>0, \quad p(x)>0 .
$$

In the theory of equation (8.1) an important role is played by the quantity

$$
\phi_{y}(x)=r(x) y^{\prime}(x) y^{\prime \prime}(x)-y(x)\left[r(x) y^{\prime \prime}(x)\right]^{\prime} .
$$

If $y(x)$ is a solution of (8.1),

$$
\phi_{\nu}^{\prime}(x)=r y^{\prime \prime 2}-y\left(r y^{\prime \prime}\right)^{\prime \prime}=r y^{\prime \prime 2}+p y^{2},
$$

and, therefore,

$$
\phi_{y}(b)-\phi_{y}(a)=\int_{a}^{b}\left(r y^{\prime \prime 2}+p y^{2}\right) d x .
$$

Since the right-hand side of (8.3) is positive if $b>a,(8.3)$ implies the following result.

Lemma 8.1. If $y(x)$ is a nontrivial solution of (8.1), the quantity (8.2) is strictly increasing with increasing $x$.

In particular, $\phi_{y}(x)$ can vanish but once. Since $\phi_{y}(x)$ will vanish if $y(x)$ has a double zero, it follows that no solution of (8.1) can have more than one double zero. For convenient reference we state this as a separate lemma.

Lemma 8.2. No nontrivial solution of (8.1) can have more than one double zero.

It will be seen later that a central role is played by the solutions of (8.1) which have a triple zero at a given point $x=a$. These solutions will be called principal solutions, and they will be normalized in the following manner.

Definition 8.3. The principal solution $y(x)=y(x, a)$ of (8.1) is defined by the conditions

$$
y(a)=y^{\prime}(a)=y^{\prime \prime}(a)=0, \quad r(a) y^{\prime \prime \prime}(a)=1 .
$$


We remark here that it is possible to define three additional types of principal solutions associated with the point $x=a$ by requiring that any three of the four quantities $y, y^{\prime}, r y^{\prime \prime},\left(r y^{\prime \prime}\right) !$ vanish at $x=a$ and that the fourth be equal to 1 . Their properties are analogous to those of $y(x, a)$, and they may be utilized for the investigation of the general properties of (8.1) in much the same way as $y(x, a)$. However, since this would not involve any new principles, we shall confine ourselves to those aspects of equation (8.1) which depend on the principal solutions $y(x, a)$.

The following symmetry property of $y(x, a)$ is easily proved.

Lemma 8.4. If $y(x, a)$ denotes a principal solution of (8.1), then

$$
y(x, a)=-y(a, x) .
$$

We set $u(x)=y(x, a)$ and $v(x)=y(x, b)$, where $b \neq a$. Both $u(x)$ and $v(x)$ are solutions of $(8.1)$, and we have therefore $v\left(r u^{\prime \prime}\right)^{\prime \prime}-u\left(r v^{\prime \prime}\right)^{\prime \prime}=0$. Integrating this we obtain

$$
v\left(r u^{\prime \prime}\right)^{\prime}-u\left(r v^{\prime \prime}\right)^{\prime}-r\left(v^{\prime} u^{\prime \prime}-u^{\prime} v^{\prime \prime}\right)=c=\text { const. }
$$

Setting $x=a$ and $x=b$, respectively, and observing conditions (8.4) we find that $v(a)=c=-u(b)$. Hence, $y(b, a)=-y(a, b)$, and Lemma 8.4 follows.

2. It will be shown in the following section that the zeros of $y(x, a)$ in $(a, \infty)$ play a role in the theory of equation (8.1) which is in many ways similar to that of the conjugate points $\eta_{\nu}(a)$ used in the discussion of equation (2.1). We shall therefore also speak of conjugate points in the present case.

Definition 8.5. If $\eta_{1}(a), \eta_{2}(a), \cdots\left(a<\eta_{1}<\eta_{2}<\cdots\right)$ are the zeros of the principal solution $y(x, a)$ in $(a, \infty)$, then $x=\eta_{n}(a)$ will be called the nth conjugate point of $x=a$.

The conjugate points have the following monotonicity property.

THEOREM 8.6. If $b>a$ and $x=\eta_{n}(a)$ is the nth conjugate point of $x=a$, then (8.7)

$$
\eta_{n}(b)>\eta_{n}(a) \text {. }
$$

It should be noted that (8.7) also covers the case in which $\eta_{n}(b)=\infty$, that is, the case in which there does not exist a conjugate point of order $n$.

We first prove (8.7) for $n=1$. According to (8.4), the principal solution $y(x, a)$ is positive in the interval $\left(a, \eta_{1}(a)\right)$, and $y(x, b)$ is positive in $\left(b, \eta_{1}(b)\right)$. If we had $\eta_{1}(b)<\eta_{1}(a), y(x, a)$ would be positive in the closed interval $\left[b, \eta_{1}(b)\right]$, and it would follow from Lemma 1.2 that there exists a constant $\mu$ such that $u(x)=y(x, a)-\mu y(x, b)$ has a double zero somewhere in $\left(b, \eta_{1}(b)\right)$. Since both $y(x, a)$ and $y(x, b)$ are positive in the open interval, we have, moreover, $\mu>0$. We now consider the quantity $\phi_{u}(x)$ defined by (8.2). Since $\phi_{u}(x)$ vanishes at the double zero of $u(x)$, and $u(x)$ is a solution of (8.1), it follows from Lemma 8.1 that $\phi_{u}(b)<0$. Setting $y(x, a)=y_{1}(x)$ and $y(x, b)=y_{2}(x)$ we have, because of the conditions $(8.4), \phi_{u}(b)=\phi_{\nu_{1}}(b)+\mu y(b, a)$. But this implies 
a contradiction, since all the quantities on the right-hand side are positive. Indeed, $y(b, a)$ is positive by assumption, $\mu$ was just shown to be positive, and $\phi_{y_{1}}(b)$ is positive by virtue of Lemma 8.1 and the fact that $\phi_{y_{1}}(a)=0$. We have thus proved (8.7) for $n=1$.

A similar argument will also dispose of the general case if we make use of the fact-to be proved presently - that $\eta_{n}(a)$ is a continuous function of $a$. If we take $b$ such that $0<b-a<\epsilon$, where $\epsilon$ is small, $\eta_{n}(b)$ will be close to $\eta_{n}(a)$. We, moreover, take $\epsilon$ small enough so as to insure that $\eta_{\nu}(b)>\eta_{\nu-1}(a)$ for $\nu=2,3, \cdots, n$. We now suppose that (8.7) does not hold for all $\nu$ such that $\nu \leqq n$. Since, as we have just seen, it does hold for $\nu=1$, there will exist an integer $m(2 \leqq m \leqq n)$ such that $\eta_{\nu}(a)<\eta_{\nu}(b)$ for $1 \leqq \nu<m$, and $\eta_{m}(a)>\eta_{m}(b)$. Because of our choice of $\epsilon$ we shall then have $\eta_{m-1}(a)<\eta_{m-1}(b)<\eta_{m}(b)<\eta_{m}(a)$. Since (by Lemma 8.2) the zeros of $y(x, a)$ in $(a, \infty)$ are simple, $y(x, a)$ changes its sign if $x$ passes through a conjugate point. Hence, the sign of $y(x, a)$ in $\left(\eta_{m-1}(a), \eta_{m}(a)\right)$ will be the same as the sign of $y(x, b)$ in $\left(\eta_{m-1}(b), \eta_{m}(b)\right)$, and we may conclude from Lemma 2.1 that there exists a positive number $\mu$ such that $u(x)=y(x, a)-\mu y(x, b)$ has a double zero somewhere in $\left(\eta_{m-1}(b)\right.$, $\left.\eta_{m}(b)\right)$. At this point the function $\phi_{u}(x)$ will vanish, and it follows from Lemma 8.1 that $\phi_{u}(b)<0$. But, as shown above, $\phi_{u}(b)>0$ if $a<b<\eta_{1}(a)$, and we have arrived at a contradiction. The proof of Theorem 8.6 will thus be complete if we prove the continuity of $\eta_{n}(a)$ as a function of $a$. For convenient reference we formulate this property as a separate lemma.

Lemma 8.7. If $x=\eta_{n}(a)$ is the nth conjugate point of $x=a$ with respect to equation (8.1), $\eta_{n}(a)$ is a continuous function of $a$.

We first observe that, by virtue of $(8.5), y(x, a)$ is a continuous function of the variable $a$ and, in fact, has as many derivatives with respect to $a$ as it has with respect to $x$. If $n$ is a positive integer, we denote by $M$ the smallest maximum which the function $\xi=|y(x, a)|$ takes in any sub-intervals of $\left(a, \eta_{n}(a)\right)$ which are bounded by two consecutive conjugate points of $x=a$. Since $y(x, a)$ is continuous in $a$, we shall have $|y(x, b)-y(x, a)|<\epsilon<M$, provided $|b-a|$ is taken small enough, and it follows that $y(x, b)$ is of the same sign as $y(x, a)$ at those points of each arch of the curve $\xi=y(x, a)$ at which $|y(x, a)|$ attains its maximum and, of course, also in certain intervals containing these points. Because of the continuity of $y(x, a)$ as a function of $x$, the latter intervals will approximate the intervals $\left(\eta_{\nu}(a), \eta_{\nu+1}(a)\right)$ arbitrarily closely, provided $\epsilon$ is taken small enough. If $|b-a|<\delta(\epsilon)$, the zeros of $y(x, b)$ will therefore lie in arbitrarily small intervals containing the zeros of $y(x, a)$. This completes the proof of Lemma 8.7.

As an application of Theorem 8.6 and Lemma 8.7 we derive a result which will be needed in the following section.

LEMMA 8.8. If the point $x=b$ coincides with a zero of $y(x, a)$ in $(a, \infty)$, then $y(x, a)$ and $y(x, b)$ have the same number of zeros in $[a, b]$. 
We note that, by Lemma $8.4, x=a$ is a zero of $y(x, b)$. Lemma 8.8 will therefore remain true if $[a, b]$ is replaced by the open interval $(a, b)$. To prove Lemma 8.8 we suppose that $x=b$ is the $n$th zero of $y(x, a)$ in $(a, \infty)$, that is, $b=\eta_{n}(a)$. If $a$ increases continuously in $(a, b)$, it follows from Lemma 8.7 and Theorem 8.6 that the $n-1$ zeros of $y(x, a)$ in $(a, b)$ move continuously to the right, and it is clear that each of these zeros will finally pass through the point $x=b$. There will thus exist $n-1$ points $a_{1}, \cdots, a_{n-1}\left(a<a_{1}<\cdots\right.$ $\left.<a_{n-1}<b\right)$ in $(a, b)$ such that the solution $y\left(x, a_{\nu}\right)(\nu=1, \cdots, n-1)$ has a zero at $x=b$, that is; $y\left(b, a_{v}\right)=0$. By Lemma 8.4 , we also have $y\left(a_{v}, b\right)=0$, and this shows that the principal solution $y(x, b)$ has at least $n-1$ zeros in $(a, b)$. If we reverse the roles of $y(x, a)$ and $y(x, b)$ and observe that Theorem 8.6 and Lemma 8.7 are also valid for the zeros of $y(x, a)$ in $(0, a)$ (which may be regarded as conjugate points of negative index), the result follows.

\section{Separation theorems.}

1. Simple examples show that, in general, the zeros of two solutions of (8.1) will not separate each other as in the case of a second-order equation. The pattern of distribution of the zeros shows, however, a degree of regularity which goes considerably beyond the laws of distribution characteristic of the solutions of (2.1). We begin the discussion with a few cases in which we have simple Sturmian separation.

LeMma 9.1. If $u(x)$ and $v(x)$ are essentially different nontrivial solutions of (8.1) which have a double zero at $x=a$, the zeros of $u(x)$ and $v(x)$-both in $(0, a)$ and $(a, \infty)$-separate each other.

The proof is very simple. If there exist two consecutive zeros $x=\alpha, \beta$ of, say, $u(x)$ such that $v(x) \neq 0$ in $[\alpha, \beta]$, then it follows from Lemma 1.2 that the solution $w(x)=u(x)-\mu v(x)$-with a suitable constant $\mu$-has a double zero at some point of $(\alpha, \beta)$. Since $w(x)$ also has a double zero at $x=a$, this contradicts Lemma 8.2. To show that $u(x)$ and $v(x)$ can have no common zeros other than at $x=a$, we note that, by Lemma 8.2 , such zeros are necessarily simple. By suitable choice of the constant $\mu, v(x)=u(x)-\mu v(x)$ can be made to have a double zero at such a point, and the same argument applies. We add that, in the special case $r(x)=1$, Lemma 9.1 was proved in [4].

We next prove a result which lies somewhat deeper.

Lemma 9.2. If $u(x)$ is a solution of (8.1) such that $u(a)=0$ and $\phi_{u}(a) \geqq 0$, where $\phi_{u}(x)$ is defined in (8.2), then the zeros of $u(x)$ and the principal solution $y(x, a)$ in $(a, \infty)$ separate each other.

If there is a case of nonseparation in $(a, \infty)$, we conclude as in the proof of Lemma 9.1 that there exist constants $\lambda$ and $\mu$ such that the solution $w(x)$ $=\lambda u(x)+\mu y(x, a)$ has a double zero somewhere in $(a, \infty)$. At this point, the expression $\phi_{w}(x)$ vanishes, and it follows therefore from Lemma 8.1 that $\phi_{w}(a)<0$. However, because $u(a)=0$ and conditions (8.4) hold, $\phi_{w}(a)$ reduces 
to $\lambda^{2} \phi_{u}(a)$. Since $\phi_{u}(a) \geqq 0$, this is absurd, and the result follows.

If the condition $\phi_{u}(a) \geqq 0$ is replaced by $\phi_{u}(a) \leqq 0$, the same method of proof yields a separation theorem for the interval $(0, a)$.

Lemma 9.3. If the solution $u(x)$ of (8.1) satisfies $u(a)=0$ and $\phi_{u}(a) \leqq 0$, the zeros of $u(x)$ and $y(x, a)$ in $(0, a)$ separate each other.

2. The central results of this section will be Theorems 9.5 and 9.6. Theorem 9.5 shows that the principal solutions $y(x, a)$ occupy a position in the theory of equation (8.1) which is analogous to that of the solutions $y_{\nu}(x)$ (cf. Theorem 3.6) in the theory of equation (2.1). Theorem 9.6, on the other hand, has no analogue in the theory of equation (2.1) (since (2.1) may have both oscillatory and nonoscillatory solutions which vanish at the same point) and it may serve to illustrate the fundamentally different character of the two classes of equations.

The proof of these two results has to overcome certain technical difficulties which are caused by the fact that the pattern of distribution of the zeros of a solution $y(x)$ of $(8.1)$ is different according as the quantity $\phi_{y}(x)$ defined in (8.2) is positive or negative. This pattern may become particularly irregular in the vicinity of a point at which $\phi_{y}(x)$ changes its sign (an event which, in view of Lemma 8.1, can occur only once in $(0, \infty)$ ), and such a point will therefore require special treatment.

As a first step towards the proofs of Theorems 9.5 and 9.6, we derive the following result.

Lemma 9.4. Let $u(x)$ be a nontrivial solution of (8.1) which vanishes at $x=a$ but is not a constant multiple of either of the principal solutions $y(x, a)$, $y\left(x, \eta_{1}(a)\right)$. If $\phi_{u}(x)$ is of constant sign in $\left(a, \eta_{1}(a)\right), u(x)$ must have a zero in this interval.

We first prove this lemma in the case in which $\phi_{u}(x)$ is positive. By assumption, either $u^{\prime}(a)$ or $u^{\prime \prime}(a)$ will be different from zero, and we may normalize $u(x)$ so that its first nonvanishing derivative at $x=a$ is positive. The solution $u(x)$ will then be positive between $x=a$ and its first zero in $(a, \infty)$. We set $b=\eta_{1}(a)$, and we suppose that $u(x)>0$ in $(a, b]$, contrary to the assertion of Lemma 9.4. Setting $y(x, a)=v(x)$ and $\sigma(x)=v(x) u^{\prime}(x)-u(x) v^{\prime}(x)$, we have $\sigma(b)=-u(b) v^{\prime}(b)$, and this is positive since $u(b)>0, v^{\prime}(b)<0$ (according to Lemma 8.2 , the zero of $v(x)$ at $x=b$ is simple). An elementary computation shows that $\sigma(a)=\sigma^{\prime}(a)=\sigma^{\prime \prime}(a)=0, \sigma^{\prime \prime \prime}(a)=-2 u^{\prime}(a) v^{\prime \prime \prime}(a)$, and this is negative if $u^{\prime}(a)>0$. Should $u^{\prime}(a)$ vanish, then an additional differentiation will yield $\sigma^{\mathrm{iv}}(a)=-2 u^{\prime \prime}(a) v^{\prime \prime \prime}(a)$, and this is again negative (we have used the fact that, by $(8.4), v^{\prime \prime \prime}(a)>0$ and that $v(x)>0$ in $\left.(a, b)\right)$. It follows that in all cases $\sigma(x)$ will be negative for values $x$ slightly larger than $a$. Since $\sigma(b)>0, \sigma(x)$ must vanish at some point $x=c$ in $(a, b)$. Hence, there exist two constants $\lambda, \mu\left(\lambda^{2}+\mu^{2}>0\right)$ such that the function $w(x)=\lambda u(x)+\mu v(x)$ has a 
double zero at $x=c$. Now $w(x)$ is a solution of (8.1), and, as the definition (8.2) shows, $\phi_{w}(c)=0$. By Lemma 8.1 , we thus have $\phi_{v}(a)<0$. But $\phi_{w}(a)$ is easily seen to reduce to $\lambda^{2} \phi_{u}(a)$, and this is non-negative since $\phi_{u}(a) \geqq 0$. The assumption that $u(x)>0$ in $(a, b]$ will therefore lead to a contradiction.

It remains to be shown that the first zero of $u(x)$ in $(a, \infty)$ cannot coincide with the point $x=b$. If there exists a zero of $u(x)$ at $x=b$, it is necessarily simple, since otherwise we would have $\phi_{u}(b)=0$, and thus by Lemma 8.1, $\phi_{u}(x)<0$ in $(a, b)$, contrary to hypothesis. Since $u(x)>0$ in $(a, b)$ we further have $u^{\prime}(b)<0$. As $v^{\prime}(b)$ is negative for the same reason, there exists a positive constant $\mu$ such that the solution $w(x)=u(x)-\mu v(x)$ has a double zero at $x=b$. Hence, $\phi_{w}(b)=0$, and a contradiction is arrived at in the same way as before.

We now turn to the case in which $\phi_{u}(x)$ is negative in $(a, b)$. Again, we assume that $u(x)>0$ in $(a, b)$. By (8.5), the principal solution $y(x, b)$ vanishes at $x=a$. By Lemma 8.8 and Definition $8.3, y(x, b)$ is negative in $(a, b)$. Since $\phi_{u}(x)<0$ in $(a, b)$ the zero of $u(x)$ at $x=a$ is simple, and we accordingly have $u^{\prime}(a)>0$. Hence, there exists a positive constant $\mu$ such that $w(x)=u(x)$ $+\mu y(x, b)$ has a double zero at $x=a$. It follows that $\phi_{w}(a)=0$ and thus, by Lemma 8.1, $\phi_{w}(b)>0$. But $\phi_{w}(b)$ is easily seen to reduce to $\phi_{u}(b)-\mu u(b)$, and this cannot be positive since $\phi_{u}(b) \leqq 0$ and $u(b) \geqq 0$. A contradiction can be avoided only if $w(x)$ vanishes identically; that is, if $u(x)$ is a constant multiple of $y(x, b)$. This completes the proof of Lemma 9.4.

It is worth noting that the conclusion of Lemma 9.4 does not necessarily hold if $\phi_{\boldsymbol{u}}(x)$ changes its sign in $\left(a, \eta_{1}(a)\right)$, as the following example shows. If we construct a solution $v(x)$ of (8.1) which vanishes at $x=a$ and has a double zero at some point of $(a, b)$-where $b=\eta_{1}(a)$-it follows from Theorem 9.5 that the zero of $v(x)$ at $x=a$ is simple and that there are no more zeros in $(a, b]$. Hence, $v(x)$ does not change its sign in $(a, b)$, and it may be assumed to be non-negative there. If $\mu$ is a positive constant, the solution $w(x)=y(x, a)$ $+\mu v(x)$ will accordingly be positive in $(a, b]$. Since this would contradict the conclusion of Lemma 9.4 if $\phi_{w}(x)$ had a constant sign in $(a, b), \phi_{w}(x)$ must vanish somewhere in this interval. It is also not difficult to confirm by direct computation that $\phi_{w}(a)<0$ and $\phi_{w}(b)>0$.

This example shows that the vanishing of the expression $\phi_{u}(x)$ may disturb the regular pattern of distribution of the zeros of a solution $u(x)$. This disturbance is, however, not too serious since it may be shown that the conclusion of Lemma 9.4 is valid regardless of the nature of $\phi_{u}(x)$, if $\eta_{1}(a)$ is replaced by the second conjugate point $\eta_{2}(a)$. The effect of the change of sign of $\phi_{u}(x)$-and it should be remembered that, according to Lemma 8.1, there can be only one such change of sign-will thus be largely of a local character. At a zero of $\phi_{u}(x)$ the solution $u(x)$ may, roughly speaking, "skip" a change of sign. This will result either in a zero being left out, or in the appearance of a double zero. We do not prove here this extension of Lemma 9.4 to the gen- 
eral case since it will be obtained as a special case of Theorem 9.6.

3. The principal results of this section are contained in the following two theorems.

THEOREM 9.5. Let $b=\eta_{n}(a)$ denote the nth conjugate point of $x=a$. If $u(x)$ is a nontrivial solution of (8.1) which vanishes at $x=a$, the number of zeros of $u(x)$ in $[a, b]$ is smaller than the number of zeros of either of the principal solutions $y(x, a), y(x, b)$, unless $u(x)$ is a constant multiple of $y(x, a)$ or $y(x, b)$.

THEOREM. 9.6. Let $b=\eta_{n}(a)$ denote the nth conjugate point of $x=a$, and let $\eta_{n}(a)$ be finite. If $u(x)$ is a nontrivial solution of (8.1) which vanishes at $x=a$, $u(x)$ has at least $n$ zeros in $[a, b)$. If, in addition, $\phi_{u}(x)$ does not change its sign in this interval, the number of zeros of $u(x)$ in $[a, b)$ is at least $n+1$.

We first prove Theorem 9.5 in the case in which $\phi_{u}(a) \geqq 0$. If we combine Lemma 9.2 with Lemma 9.4, we see that although the first zero of $u(x)$ in $(a, \infty)$ precedes that of $y(x, a)$ in the same interval, the multiplicity of the zero of $u(x)$ at $x=a$ is lower than that of the zero of $y(x, a)$ at the same point. The $n$th zero of $y(x, a)$ will therefore always precede the $n$th zero of $u(x)$ in $[a, \infty)$, and the result follows. The same considerations also prove the second part of Theorem 9.6 in the case in which $\phi_{u}(a) \geqq 0$. Indeed, according as $u^{\prime}(a) \neq 0$ or $u^{\prime}(a)=0, u(x)$ will have, respectively, two or three zeros in $\left(a, \eta_{1}(a)\right)$. Since, by Lemma 9.2, the interval between two consecutive conjugate points contains precisely one zero of $u(x)$, there will be either $n+1$ or $n+2$ zeros in $[a, b)$.

The case in which $\phi_{u}(b) \leqq 0$-and therefore $\phi_{u}(x)<0$ in $(a, b)$-is dealt with in a similar manner. According to Lemma 9.3, the zeros of $u(x)$ and $y(x, b)$ in $(a, b)$ separate each other. Lemma 9.4 remains valid if $\eta_{1}(a)$ is replaced by $\eta_{-1}(a)$, that is the last zero of $y(x, a)$ in $(0, a)$. This may be seen either by making the requisite trivial modifications in the proof of Lemma 9.4, or else by applying Lemma 9.4 to the equation obtained from (8.1) by substituting $2 a-x$ for $x$. The zeros of $u(x)$ may therefore be counted in the same way as before, and the result follows.

We now prove Theorem 9.5 for the case in which $\phi_{u}(x)$ changes it sign at a point $x=c(a<c<b)$. We first assume that $x=c$ is not a double zero of $u(x)$, and we denote by $x=\alpha$ and $x=\beta(a \leqq \alpha<\beta \leqq b)$ the two consecutive zeros of $u(x)$ (one of which may coincide with $x=c$ ) between which $x=c$ is located. Since $\phi_{u}(\beta) \geqq 0$, we may conclude from Lemmas 9.2 and 9.3 that $u(x)$ has at most one more zero in $(\beta, b]$ than $y(x, \beta)$. Similarly, Lemma 9.3 and the modification of Lemma 9.4 just mentioned show that $u(x)$ has at most one more zero in $[a, \alpha)$ than $y(x, \alpha)$, if it is observed that $\phi_{u}(\alpha) \leqq 0$. According to Theorem 8.6 and Lemma 8.7, the zeros of $y(x, a)$ move continuously to the right if $a$ increases, and to the left if $a$ decreases. The same is true of the zeros of $y(x, a)$ in $(0, a)$ - the conjugate points of negative index-since, by Lemmas 
8.4 and 8.8, the point $x=a$ is the $n$th conjugate point of $x=\eta_{-n}(a)$. If $x=\delta$ is any point such that $\alpha \leqq \delta \leqq \beta$, we may therefore conclude that $y(x, \delta)$ does not have fewer zeros in $(\delta, b]$ than $y(x, \beta)$ has in $(\beta, b]$, and not fewer zeros in $[a, \delta)$ than $y(x, \alpha)$ has in $[a, \alpha)$. If we take into account the triple zero of $y(x, \delta)$ at $x=\delta$ and the simple zeros of $u(x)$ at $x=\alpha$ and $x=\beta$, we thus find that the number of zeros of $u(x)$ in $[a, b]$ cannot exceed the number of zeros of $y(x, \delta)$ in this interval by more than one.

We proceed to show that $y(x, \delta)$ has fewer zeros in $[a, b]$ than $y(x, a)$. If $x=\delta$ coincides with one of the conjugate points of $x=a$ in $(a, b)$-say with $\eta_{\nu}(a)$ - the principal solutions $y(x, a)$ and $y(x, \delta)$ have, by Lemma 8.8 , the same number of zeros in $[a, \delta]$. Since, by Lemmas 9.2 and 9.4 , the zeros of these solutions in $(\delta, b)$ separate each other, and $y(x, a)$ has both the first and the last zero in $(\delta, b], y(x, a)$ is thus found to have one more zero in $[a, b]$ than $y(x, \delta)$. We now let $\delta$ vary continuously from $\eta_{\nu}(a)$ to $\eta_{\nu+1}(a)$, and we use Theorem 8.6 and Lemma 8.7. In this process, no zeros of $y(x, \delta)$ can cross the point $x=a$, and the number of zeros of $y(x, \delta)$ in $(\delta, b]$ cannot increase. The number of zeros of $y(x, \delta)$ in $[a, b]$ is therefore less than the number of zeros of $y(x, a)$ in this interval if $\delta$ is any number such that $a<\delta<b$. If we combine this with the relation between the zeros of $u(x)$ and $y(x, \delta)$ found above, we arrive at the result that $u(x)$ has no more zeros in $[a, b]$ than $y(x, a)$.

If $u(x)$ has a double zero at $x=c$, we compare the zeros of $u(x)$ with those of $y(x, c)$. Lemmas 9.1 and 9.4 show that $u(x)$ has at most one more zero in $[a, b]$ than $y(x, c)$. It follows therefore as before that $u(x)$ cannot have more zeros in $[a, b]$ than $y(x, a)$.

It remains to be shown that the number of zeros of $u(x)$ in $[a, b]$ is actually less than that of $y(x, a)$ unless $u(x)$ coincides with a constant multiple of $y(x, a)$ or $y(x, b)$. To this end we assume that there exists a solution $u(x)$ which vanishes at $x=a$ and $x=b\left(b=\eta_{n}(a)\right)$ and which has precisely as many zeros in $[a, b]$ as $y(x, a)$. It is easy to see that $u(x)$ must be of the form $A y(x, a)+B y(x, b)$, where $A$ and $B$ are constants. If this were not the case, it would be possible to represent any solution of (8.1) which vanishes at $x=a$ as a linear combination of $u(x)$ and the two principal solutions in question. Since all these solutions are zero at $x=b$, it follows that any solution of (8.1) which vanishes at $x=a$ must likewise vanish at $x=b$. To show that this is absurd it is sufficient to consider a solution $v(x)$ of (8.1) for which $v(a)$ $=v^{\prime}(a)=0, v^{\prime \prime}(a) \neq 0$, and to refer to Lemma 9.1. Hence, $u(x)=A y(x, a)$ $+B y(x, b)$.

According to Lemma 8.8 , the solutions $y(x, a)$ and $y(x, b)$ have the same number of zeros in $(a, b)$. We now need the following more precise information.

LEMMA 9.7. If $x=b$ coincides with a zero of $y(x, a)$, then the zeros of $y(x, a)$ and $y(x, b)$ in $(a, b)$ separate each other. 
Indeed, if $x=c$ is a zero of $y(x, a)$ in $(a, b)$, the principal solution $y(x, c)$ has a zero at $x=a$ and, by Lemma 9.2, $y(x, c)$ has as many zeros in $(c, b)$ as $y(x, a)$. If $x=c^{\prime}$ is the zero of $y(x, a)$ immediately following its zero at $x=c$, $y\left(x, c^{\prime}\right)$ will therefore have one zero less in $\left(c^{\prime}, b\right)$ than it had in $(c, b)$. Since, by Theorem 8.6 and Lemma 8.7 , the zeros of $y(x, c)$ increase continuously with $x$, there must exist a value $c^{\prime \prime}$ such that $c<c^{\prime \prime}<c^{\prime}$, and such that $y\left(x, c^{\prime \prime}\right)$ vanishes at $x=b$. Since $c^{\prime \prime}$ is, by Lemma 8.4, a zero of $y(x, b)$, it follows therefore that between two consecutive zeros of $y(x, a)$ there must be a zero of $y(x, b)$. If the roles of $y(x, a)$ and $y(x, b)$ are interchanged, the result follows.

We conclude from Lemma 9.7 that the curves $\xi=A y(x, a)$ and $\xi$ $=-B y(x, b)$ must intersect in each interval bounded by two consecutive zeros of $y(x, a)$ in $(a, b)$. By Lemma 9.4, they have, moreover, a point of intersection in each of the intervals bounded by $x=a$ and the first zero, and by $x=b$ and the last zero, respectively. If $b=\eta_{n}(a)$, this will thus account for $n$ zeros of $u(x)=A y(x, a)+B y(x, b)$. Adding to this the two simple zeros of $u(x)$ at $x=a$ and $x=b$, we obtain a total of $n+2$ zeros in $[a, b]$. Additional zeros of $u(x)$ could conceivably be brought about by the two curves having more than one point of intersection between two consecutive zeros of $y(x, a)$. Since, however, the zeros of $y(x, a)$ and $y(x, b)$ separate each other, the number of the points of intersection in such an interval is necessarily odd. The number of zeros of $u(x)$ in $[a, b]$ is therefore either $n+2$ or at least $n+4$. Since, however, the number of zeros of $y(x, a)$ in $[a, b]$ is $n+3$, the latter alternative is excluded. This completes the proof of Theorem 9.5.

We now turn to the proof of Theorem 9.6, and we recall that the only case not yet dealt with was that in which $\phi_{u}(x)$ changes its sign in the interval considered. We accordingly assume that $u(a)=u(b)=0, \phi_{u}(c)=0(a<c<b)$, and that $\eta_{n-1}(a) \leqq b<\eta_{n}(a)$. Theorem 9.6 will be proved if we can show that $u(x)$ has at least $n$ zeros in $[a, b]$.

If $n_{1}$ and $m_{1}$ denote, respectively, the number of zeros of $y(x, a)$ and $u(x)$ in $(a, c]$, it follows from Lemmas 9.2 and 9.4 that $m_{1} \geqq n_{1}$. Similarly, if $n_{2}$ and $m_{2}$ are, respectively, the number of zeros of $y(x, b)$ and $u(x)$ in $(c, b)$, we have $m_{2} \geqq n_{2}$. If $n_{3}$ stands for the number of zeros of $y\left(x, \eta_{n-1}(a)\right)$ in $\left(c, \eta_{n-1}(a)\right)$, it follows from Theorem 8.6 that $n_{3} \leqq n_{2}$. Hence, $m_{1}+m_{2} \geqq n_{1}+n_{2} \geqq n_{1}+n_{3}$. Lemma 9.7 shows, on the other hand, that the value of $n_{1}+n_{3}$ is either $n-1$ or $n-2$. Therefore, $m_{1}+m_{2} \geqq n-2$. If we add to this the zeros of $u(x)$ at $x=a$ and $x=b$, we thus find that altogether there are at least $n$ zeros of $u(x)$ in $[a, b]$ This proves Theorem 9.6.

4. Theorems 9.5 and 9.6 provide bounds for the number of zeros which a solution of (8.1) which vanishes at $x=a$ may have in an interval bounded by $x=a$ and a conjugate point $x=\eta_{n}(a)$. Combining these two results, we can obtain an estimate for the largest possible difference between the number of zeros of two different solutions in an interval which is not necessarily bounded by conjugate points. 
THEOREM 9.8. Let $u(x)$ and $v(x)$ be two nontrivial solutions of (8.1) which vanish at $x=a$. If $b>a$, the number of zeros of $u(x)$ in $[a, b]$ cannot differ from the number of zeros of $v(x)$ in this interval by more than three.

Indeed, if $\eta_{n}(a) \leqq b<\eta_{n+1}(a)$, it follows from Theorem 9.5 that both $u(x)$ and $v(x)$ have not more than $n+3$ zeros in $[a, b]$. Since, by Theorem 9.6, both these solutions have at least $n$ zeros in this interval, the result follows. We add that the difference between the number of zeros of $u(x)$ and $v(x)$ in $[a, b]$ may actually amount to 3 . The principal solution $y(x, a)$ has four zeros in $\left[a, \eta_{1}(a)\right]$ and, as shown above there exist solutions of $(8.1)$ which have a simple zero at $x=a$ and do not vanish in $\left(a, \eta_{1}(a)\right]$.

If we free ourselves from the assumption that $u(x)$ and $v(x)$ should have a common zero, then the difference between the number of zeros these solutions may have in any given closed interval may increase by an additional unit.

Theorem 9.9. If $u(x)$ and $v(x)$ are two nontrivial solutions of (8.1), the number of zeros of $u(x)$ in any closed interval $[a, b]$ cannot differ from the number of zeros of $v(x)$ in $[a, b]$ by more than four.

We may assume that $x=a$ is a zero of one of these solutions, say $u(x)$. We denote by $x=a^{\prime}$ the first zero of $v(x)$ in $[a, b]$, and we first assume that $\phi_{v}(x)$ does not change its sign in $\left(a, a^{\prime}\right)$. By Lemma 9.4, the first conjugate point of $x=a$ cannot lie in $\left(a, a^{\prime}\right]$. The principal solution $y(x, a)$ can therefore-in view of Theorem 8.6 and Lemma 9.2-have at most one more conjugate point in $[a, b]$ than $y\left(x, a^{\prime}\right)$, and it follows from the considerations which led to Theorem 9.8, that the number of zeros of one solution cannot exceed that of the other solution by more than four. If $\phi_{v}(x)$ does change its sign in $\left(a, a^{\prime}\right)$, we can-in accordance with Theorem 9.6-conclude only that the second conjugate point of $x=a$ does not lie in $\left(a, a^{\prime}\right]$. This may seem to give rise to the possibility of the zeros of $u(x)$ exceeding those of $v(x)$ by five. However, if $\phi_{v}(x)$ changes its sign in $\left(a, a^{\prime}\right)$, then $\phi_{v}(x)>0$ in $\left[a^{\prime}, b\right]$, and the count of the minimum number of zeros of $v(x)$ in this interval may be based on the sharper estimate given in the second part of Theorem 9.6. This completes the proof. We note that Theorem 9.9 is again the best possible of its kind, as may be seen from the example used to show the sharpness of Theorem 9.8. If $u(x)$ is such that $u(a)=0$ and $u(x) \neq 0$ in $\left(a, \eta_{1}(a)\right]$ and $\epsilon$ is a sufficiently small positive constant, $u(x)$ will have no zeros in $\left[a+\epsilon, \eta_{1}(a+\epsilon)\right]$, while $y(x, a+\epsilon)$ has four zeros in this interval.

Theorem 9.9 has the following immediate corollary.

COROLlaRY 9.10. The solutions of (8.1) are either all oscillatory or all nonoscillatory.

As already pointed out in $\S 1$, this result shows how deeply the behavior of the solutions of equation (8.1) differs from that of the solutions of (2.1). 
As we saw in $\S 2$, the same equation (2.1) may have both oscillatory and nonoscillatory solutions. In addition to Corollary 9.10, Theorem 9.9 moreover permits the conclusion that no solution of (8.1) can have more than four zeros in $(a, \infty)$ if there exists another solution which does not vanish in this interval. If, in addition, the nonvanishing solution $y(x)$ is such that $\phi_{y}(x)$ does not change its sign in $(a, \infty)$, the following more accurate information can be obtained.

THEOREM 9.11. If there exists a solution of (8.1) such that neither $y(x)$ nor $\phi_{y}(x)$ vanishes in $(a, \infty)$, then all solutions of (8.1) are nonoscillatory in this interval; that is, no solution can have more than three zeros in $(a, \infty)$.

We suppose that there exists a solution $u(x)$ which has four zeros in $(a, \infty)$, and we denote the first and fourth zero by $x=b$ and $x=c$, respectively. By Theorem 9.5, we have $b<\eta_{1}(b) \leqq c$ and $b \leqq \eta_{-1}(c)<c$. It follows therefore from Lemma 1.2 that there exist positive constants $\lambda$ and $\mu$ such that both $u(x)=y(x)-\lambda y(x, b)$ and $v(x)=y(x)+\mu y(x, c)$ have a double zero in $(b, c)$, provided $y(x)$ is so normalized that $y(x)>0$ in $(a, \infty)$. It follows from (8.2) and (8.4) that $\phi_{u}(b)=\phi_{y}(b)+\lambda y(b)$ and $\phi_{v}(c)=\phi_{y}(c)-\mu y(c)$. Since $\phi_{u}(x)$ and $\phi_{v}(x)$ vanish at the respective double zeros of $u(x)$ and $v(x)$, we have $\phi_{u}(b)<0$ and $\phi_{v}(c)>0$. If $\phi_{y}(b)$ is positive, the resulting inequality $\phi_{\nu}(b)+\lambda y(b)<0$ implies a contradiction, and if $\phi_{\nu}(c)$ is negative, the required contradiction is obtained from $\phi_{\nu}(c)-\mu y(c)<0$. This completes the proof.

Theorem 9.11 may be applied, in particular, to a solution $y(x)=y(x, \alpha)$, where $\alpha$ is an arbitrary positive number. If (8.1) is nonoscillatory, $y(x, \alpha)$ will have a last zero, say $x=a$. Since $\phi_{y}(a)>0$, Theorem 9.11 applies, and we may conclude that no solution of $(8.1)$ has more than three zeros in $(a, \infty)$. This establishes the following result.

Lemma 9.12. If equation (8.1) is nonoscillatory, there exists a positive number a such that $(8.1)$ is nonoscillatory in $(a, \infty)$.

10. Comparison theorems. In this section we compare the oscillatory behavior of the equation

$$
\left(r(x) y^{\prime \prime}\right)^{\prime \prime}+p(x) y=0
$$

with that of the equations

$$
\left(r(x) y^{\prime \prime}\right)^{\prime \prime}+p_{1}(x) y=0
$$

and

$$
\left(r_{1}(x) y^{\prime \prime}\right)^{\prime \prime}+p(x) y=0
$$

respectively, where

$$
p_{1}(x) \geqq p(x)>0, \quad 0<r_{1}(x) \leqq r(x) .
$$


THEOREM 10.1. If $\eta_{1}(a)$ and $\eta_{1}^{\prime}(a)$ denote the first conjugate points of $x=a$ for equations (10.1) and (10.2), respectively, then

$$
\eta_{1}^{\prime}(a) \leqq \eta_{1}(a) .
$$

To prove the result, we suppose that $\eta_{1}(a)<\eta_{1}^{\prime}(a)$. If $b=\eta_{1}(a)$, it follows from Lemmas 8.4 and 8.8 that the principal solution $y(x, b)$ of $(10.1)$ vanishes at $x=a$ and is negative in $(a, b)$. We set $u(x)=y(x, b)$, and denote by $v(x)$ the principal solution of (10.2) whose triple zero is located at $x=a$. By virtue of (10.1) and (10.2), we have

$$
v\left(r u^{\prime \prime}\right)^{\prime \prime}-u\left(r v^{\prime \prime}\right)^{\prime \prime}=\left(p_{1}-p\right) u v
$$

whence

$$
\left[v\left(r u^{\prime \prime}\right)^{\prime}-u\left(r v^{\prime \prime}\right)^{\prime}-r\left(v^{\prime} u^{\prime \prime}-u^{\prime} v^{\prime \prime}\right)\right]_{a}^{b}=\int_{a}^{b}\left(p_{1}-p\right) u v d x .
$$

In view of the boundary conditions satisfied by $u(x)$ and $v(x)$, this reduces to

$$
v(b)=\int_{a}^{b}\left(p_{1}-p\right) u v d x .
$$

This, however, is absurd, since $v(x)$ is positive in $(a, b], u(x)$ is negative in $(a, b)$, and $p_{1}-p$ is non-negative. This proves Theorem 10.1 .

If the same method of proof is applied to the comparison of equations (10.1) and (10.3), the integrand on the right-hand side of (10.6) takes the form $\left(r_{1}-r\right) u^{\prime \prime} v^{\prime \prime}$, and the argument fails since both $u^{\prime \prime}(x)$ and $v^{\prime \prime}(x)$ may change their signs in the interval in question. We shall, however, establish the following comparison theorem.

THEOREM 10.2. If $\eta_{n}(a)$ and $\eta_{n}{ }^{\prime}(a)$ denote the nth conjugate points of $x=a$ for equations (10.1) and (10.3), respectively, then

$$
\eta_{1}^{\prime}(a)<\eta_{2}(a) .
$$

If $y(x)$ and $y_{1}(x)$ are solutions of (10.1) and (10.3), respectively, the functions $u(x)=r(x) y^{\prime \prime}(x)$ and $v(x)=r_{1}(x) y_{1}^{\prime \prime}(x)$ are easily seen to satisfy the equations

$$
\left(\frac{u^{\prime \prime}}{p}\right)^{\prime \prime}+\frac{u}{r}=0
$$

and

$$
\left(\frac{v^{\prime \prime}}{p}\right)^{\prime \prime}+\frac{v}{r_{1}}=0 .
$$

In view of (10.4), we may therefore conclude from Theorem 10.1 that the first 
conjugate point of $x=a$ for equation (10.9) precedes the first conjugate point of $x=a$ for equation (10.8). If the latter point is denoted by $\xi_{1}(a)$, Theorem 10.2 will thus be proved if it is shown that $\xi_{1}(a)<\eta_{2}(a)$.

If we set $y(x)=y(x, a)$, the zeros of the corresponding function $u(x)$ coincide with the points at which $y^{\prime \prime}(x, a)$ vanishes. It is easy to see that there must be a zero of $y^{\prime \prime}(x, a)$ in both $\left(a, \eta_{1}(a)\right)$ and $\left(\eta_{1}(a), \eta_{2}(a)\right)$. Indeed, if $\phi_{y}(x)$ is the expression (8.2), we have $\phi_{y}(a)=0, \phi_{y}\left(\eta_{n}\right)=r\left(\eta_{n}\right) y^{\prime}\left(\eta_{n}\right) y^{\prime \prime}\left(\eta_{n}\right)$. By Lemma 8.1, it follows that $y^{\prime}\left(\eta_{n}\right) y^{\prime \prime}\left(\eta_{n}\right)>0$. Since $y^{\prime}\left(\eta_{1}\right)<0$ and $y^{\prime}\left(\eta_{2}\right)>0$, we have $y^{\prime \prime}\left(\eta_{1}\right)<0$ and $y^{\prime \prime}\left(\eta_{2}\right)>0$. It follows that $y^{\prime \prime}(x)$ changes its sign in $\left(\eta_{1}, \eta_{2}\right)$. Since $y^{\prime \prime}(a)=0$ and $y^{\prime \prime \prime}(a)>0, y^{\prime \prime}(x)$ is positive for values slightly larger than $\dot{x}=a$. In view of $y^{\prime \prime}\left(\eta_{1}\right)<0, y^{\prime \prime}(x)$ must also vanish in $\left(a, \eta_{1}\right)$. Hence, the solution $u(x)$ of (10.8) has a zero at $x=a$ and two more zeros in $\left(a, \eta_{1}\right)$ and $\left(\eta_{1}, \eta_{2}\right)$, respectively. Since $u^{\prime \prime}=-p y$, we have $u^{\prime \prime}(a)=0$, and (8.2) shows that $\phi_{u}(a)=0$. Accordingly, $\phi_{u}(x)>0$ for $x>a$, and we may conclude from Lemmas 9.2 and 9.4 that the first conjugate point $x=\xi_{1}(a)$ separates the first and the second zeros of $u(x)$ in $(a, \infty)$. Since the second zero is contained in the interval $\left(a, \eta_{2}\right)$, this completes the proof.

It may be conjectured that the inequality (10.5) generalizes to $\eta_{n}^{\prime}(a)$ $\leqq \eta_{n}(a)$ for the conjugate points of higher order and that Theorem 10.2 has a similar generalization. We shall prove the following weaker result.

THEOREM 10.3. If $\eta_{n}(a), \eta_{n}^{\prime}(a)$, and $\eta_{n}^{\prime \prime}(a)$ denote the nth conjugate points of $x=a$ for equations (10.1), (10.2), and (10.3), respectively, then

$$
\begin{aligned}
\eta_{n}^{\prime}(a) & \leqq \eta_{2 n-1}(a), \\
\eta_{n}^{\prime \prime}(a) & <\eta_{2 n}(a) .
\end{aligned}
$$

For $n=1,(10.10)$ reduces to the assertion of Theorem 10.1. To show the validity of (10.10) for $n=2$, we apply Theorem 10.1 to the point $x=\eta_{1}(a)$. Since $\eta_{1}^{\prime}(a) \leqq \eta_{1}(a)$, it thus follows from Theorem 8.6 that the first conjugate point of $x=\eta_{1}^{\prime}(a)$ for equation (10.2) lies to the left of the first conjugate point of $x=\eta_{1}(a)$ for equation (10.1). By Lemmas 9.2 and 9.4, the latter lies to the left of the point $x=\eta_{3}(a)$, and this establishes (10.10) for $n=2$. If this argument is successively applied to the points $\eta_{3}(a), \eta_{5}(a), \cdots$, the inequality $(10.10)$ is verified for all $n$. The inequality (10.11) follows from (10.10) by the argument which led from Theorem 10.1 to Theorem 10.2.

Theorem 10.3 shows, in particular, that equations (10.2) and (10.3) will have an infinity of conjugate points of a given point $x=a$ if the same is true of equation (10.1). In view of Corollary 9.10, this implies the following result.

THEOREM 10.4. If equation (10.1) is oscillatory and the relations (10.4) hold, equations (10.2) and (10.3) are likewise oscillatory.

\section{Oscillation theorems.}

1. According to Theorem 10.4 , any equation (10.1) whose oscillatory be- 
havior is known will give rise to an oscillation criterion. The following example is typical.

THEOREM 11.1. If there exists a number $\alpha$ such that

$$
\limsup _{x \rightarrow \infty} x^{-2-\alpha} r(x)<1, \quad \liminf _{x \rightarrow \infty} 4 x^{2-\alpha} p(x)>\alpha^{2},
$$

equation (10.1) is oscillatory. If

$$
\lim _{x \rightarrow \infty} \inf x^{-2-\alpha} r(x)>1, \quad \limsup _{x \rightarrow \infty} 4 x^{2-\alpha} p(x)<\alpha^{2},
$$

the equation is nonoscillatory. The equation

$$
y^{\mathrm{iv}}+p(x) y=0 \quad(p(x)>0)
$$

is oscillatory if $\lim \inf _{x \rightarrow \infty} x^{4} p(x)>1$, and nonoscillatory if $\lim _{\sup _{x \rightarrow \infty}} x^{4} p(x)<1$.

The comparison equation on which this result depends is $\left(x^{\alpha+2} y^{\prime \prime}\right)^{\prime \prime}$ $+c^{2} x^{\alpha-2} y=0$. This equation is oscillatory if $4 c^{2}>\alpha^{2}$, and nonoscillatory if $4 c^{2} \leqq \alpha^{2}$.

2. In the remaining part of this section we restrict ourselves to the treatment of equation (11.1) in order to avoid the rather involved computations which are encountered in applying the same ideas to the general equation (10.1).

It was shown in $\S 6$ that the equation $y^{\text {iv }}-p(x) y=0$ is nonoscillatory if $x^{3} p(x)$ is summable in $(0, \infty)$. As the following result shows, the same condition will also insure the nonoscillation of equation (11.1) and it will, moreover, determine the asymptotic character of certain solutions.

THEOREM 11.2. Equation (11.1) will have a solution $y(x)$ such that

$$
\lim x^{-3}|y(x)|=c \quad(0<c<\infty)
$$

for $x \rightarrow \infty$, if, and only if,

$$
\int_{a}^{\infty} x^{3} p(x) d x<\infty \quad(0<a<\infty) .
$$

We first show that condition (11.2) is sufficient. If (11.2) holds, we may take $a$ large enough so that

$$
\int_{a}^{\infty} x^{3} p(x) d x \leqq 5
$$

Using this value of $a$ we consider the principal solution $y(x, a)$. If equation (11.1) is oscillatory, $y(x, a)$ must-in view of Corollary 9.10-have an infinity of zeros in $(a, \infty)$. If $x=b$ denotes the first of these zeros, we shall have 
$y(b, a)=0$, and $y(x, a)>0$ in $(a, b)$. Replacing (11.1) by the equivalent integral equation

$$
(x-a)^{3}=6 y(x, a)+\int_{a}^{x}(x-t)^{3} p(t) y(t, a) d t,
$$

we find that

$$
6 y(x, a) \leqq(x-a)^{3}, \quad x \in(a, b),
$$

and

$$
\begin{aligned}
(b-a)^{3} & =\int_{a}^{b}(x-t)^{3} p(t) y(t, a) d t \\
& \leqq(b-a)^{3} \int_{a}^{b} p(t) y(t, a) d t \\
& \leqq \frac{1}{6}(b-a)^{3} \int_{a}^{b}(t-a)^{3} p(t) d t .
\end{aligned}
$$

Hence,

$$
6 \leqq \int_{a}^{b}(t-a)^{3} p(t) d t<\int_{a}^{b} t^{3} p(t) d t,
$$

and this contradicts (11.3). It follows then that $y(x, a)$ cannot vanish in $(a, \infty)$; that is, $(11.1)$ is nonoscillatory.

The inequality (11.5) is thus valid for all $x$ such that $x>a$, and we may conclude from (11.4) that

$$
\begin{aligned}
(x-a)^{3} & \leqq 6 y(x, a)+(x-a)^{3} \int_{a}^{x} p(t) y(t, a) d t \\
& \leqq 6 y(x, a)+\frac{1}{6}(x-a)^{3} \int_{a}^{x}(t-a)^{3} p(t) d t,
\end{aligned}
$$

whence, in view of (11.3) and (11.5),

$$
1 / 6 \leqq 6(x-a)^{-3} y(x, a) \leqq 1 .
$$

We now need the following lemma.

Lemma 11.3. If (11.1) is nonoscillatory and $y(x)$ is a solution of (11.1) which is ultimately positive, then

$$
\lim _{x \rightarrow \infty} 6 x^{-3} y(x)=\lim _{x \rightarrow \infty} y^{\prime \prime \prime}(x) .
$$

Both limits are finite. 
To prove this result, we assume that $y(x)>0$ for $x>a$ and we again replace (11.1) by the equivalent integral equation

$$
6 R(x)=6 y(x)+\int_{a}^{x}(x-t)^{3} p(t) y(t) d t,
$$

where $R(x)$ is the polynomial

$$
R(x)=\sum_{\nu=0}^{3} \frac{y^{(\nu)}(a)}{\nu !}(x-a)^{\nu} .
$$

Since $y(x)>0$ for $x>a,(11.7)$ yields the inequality

$$
\begin{aligned}
6 R(x) & \leqq 6 y(x)+(x-a)^{3} \int_{a}^{x} p(t) y(t) d t \\
& =6 y(x)+(x-a)^{3}\left[y^{\prime \prime \prime}(a)-y^{\prime \prime \prime}(x)\right],
\end{aligned}
$$

where (11.1) has been used in the last step. By (11.8), $6(x-a)^{-3} R(x)$ tends to $y^{\prime \prime \prime}(a)$ as $x \rightarrow \infty$. The limit of $y^{\prime \prime \prime}(x)$ as $x \rightarrow \infty$ exists since, by (11.1), $y^{\prime \prime \prime}(x)$ decreases with increasing $x$. If $x$ tends to infinity through a sequence of points for which $(x-a)^{-3} y(x)$ approaches its lower limit, we have therefore

$$
\lim _{x \rightarrow \infty} y^{\prime \prime \prime}(x) \leqq \liminf _{x \rightarrow \infty} 6(x-a)^{-8} y(x) .
$$

We now choose a value $\xi$ such that $a<\xi<x$, and estimate the right-hand side of (11.7) from below. We obtain

$$
\begin{aligned}
6 R(x) & \geqq 6 y(x)+\int_{a}^{\xi}(x-t)^{3} p(t) y(t) d t \\
& \geqq 6 y(x)+(x-\xi)^{3} \int_{a}^{\xi} p(t) y(t) d t \\
& =6 y(x)+(x-\xi)^{3}\left[y^{\prime \prime \prime}(a)-y^{\prime \prime \prime}(\xi)\right] .
\end{aligned}
$$

We divide through by $(x-a)^{3}$, and let $x \rightarrow \infty$ through a sequence of points for which $(x-a)^{-3} y(x)$ tends to its upper limit, while $\xi$ is kept fixed. In view of (11.8), this leads to

$$
\limsup _{x \rightarrow \infty} 6(x-a)^{-3} y(x) \leqq y^{\prime \prime \prime}(\xi) .
$$

Since this is true for all $\xi$ such that $\xi>a$, and $y^{\prime \prime \prime}(\xi)$ decreases with increasing $\xi$, we obtain

$$
\limsup _{x \rightarrow \infty} 6(x-a)^{-3} y(x) \leqq \lim _{x \rightarrow \infty} y^{\prime \prime \prime}(x) .
$$

Iu view of (11.9), this proves Lemma 11.3.

Returning now to the proof of Theorem 11.2, we recall that, under the 
assumption (11.3), the principal solution $y(x, a)$ was shown to satisfy the inequalities (11.6). By Lemma 11.3, $\lim x^{-3} y(x, a)$ for $x \rightarrow \infty$ will therefore exist and be positive. Condition (11.2) is thus found to be sufficient for the existence of a solution with the prescribed asymptotic behavior.

To prove the necessity, we assume that (11.1) has a solution $y(x)$ such that $\lim _{x \rightarrow \infty} x^{-3} y(x)=c$, where $0<c<\infty$. Evidently, this assumption insures that $y(x)$ is ultimately nonoscillatory, since otherwise the limit in question would be zero. We may therefore assume that $y(x)$ is positive for $x>a$, where $a$ is taken large enough. It follows from Lemma 11.3 that $\lim _{x \rightarrow \infty} y^{\prime \prime \prime}(x)=6 c$. In view of (11.1), this may also be written

$$
\int_{a}^{\infty} p(x) y(x) d x=y^{\prime \prime \prime}(a)-6 c .
$$

By our assumption regarding $y(x)$, we have, furthermore, $y(x) \geqq(c-\epsilon) x^{3}$, where the positive constant $\epsilon$ may be taken arbitrarily small, provided $x$ is large enough. For sufficiently large $a$, we therefore have

$$
y^{\prime \prime \prime}(a)-6 c \geqq(c-\epsilon) \int_{a}^{\infty} x^{3} p(x) d x .
$$

This completes the proof of Theorem 11.2.

3. As another application of Lemma 11.3 we derive the following oscillation criterion.

Theorem 11.4. If

$$
\int_{a}^{\infty} x^{2} p(x) d x=\infty
$$

equation (11.1) is oscillatory.

If (11.1) is nonoscillatory, there exists-by Lemma 9.12-a value $a$ such that (11.1) is nonoscillatory in $(a, \infty)$. Accordingly, the principal solution $y(x)=y(x, a)$ will be positive for $x>a$. Equation (11.1) shows that $y^{\prime \prime \prime}(x, a)$ is decreasing for $x>a$. By Lemma $11.3, \lim y^{\prime \prime \prime}(x, a)$ for $x \rightarrow \infty$ is non-negative, and we have therefore $y^{\prime \prime \prime}(x, a)>0$ for all $x$. Hence, $y^{\prime \prime}(x, a)$ is increasing. Since $y(b, a)>0$ if $b$ is slightly larger than $a$, it follows that $y^{\prime \prime}(x, a)>A>0$, where $A$ is a suitable constant, and thus

$$
y(x, a) \geqq A(x-b)^{2}+y^{\prime}(b)(x-b)+y(b)>A(x-b)^{2} .
$$

On the other hand, we have

$$
y^{\prime \prime \prime}(b)=y^{\prime \prime \prime}(x)+\int_{b}^{x} p(t) y(t) d t>\int_{a}^{x} p(t) y(t) d t,
$$


and therefore

$$
y^{\prime \prime \prime}(b)>A \int_{b}^{x}(t-b)^{2} p(t) d t .
$$

Since the left-hand side is independent of $x$, we conclude that $(x-b)^{2} p(x)$ and therefore also $x^{2} p(x)$-is summable in $(b, \infty)$, contrary to hypothesis. This completes the proof. We remark that Theorem 11.4 strengthens an earlier result by Fite [4] according to which (11.1) is oscillatory if $p(x)$ is not summable in $(a, \infty)$.

12. Some transformations. The results of $\S \S 2-11$ refer to differential equations of the type

$$
\left(r_{1}(x) y^{\prime \prime}\right)^{\prime \prime}+P_{1}(x) y=0,
$$

where $r_{1}(x)$ is positive and $P_{1}(x)$ is of constant sign. These results can be extended to those self-adjoint fourth-order equations

$$
\left(r(x) y^{\prime \prime}\right)^{\prime \prime}+\left(q(x) y^{\prime}\right)^{\prime}+P(x) y=0
$$

which can be transformed into equations of the type (12.1), and it is therefore desirable to obtain transformations of this kind. We derive here two such transformations which do not seem to be known. Other transformations involving a very considerable amount of computational labor may be found in [6]. tion

THEOREM 12.1. Let $r(x)$ be positive and let the second-order differential equa-

$$
\left(r(x) \sigma^{\prime}\right)^{\prime}+q(x) \sigma=0
$$

be nonoscillatory in $[a, \infty)$, where $a>0$. If $\sigma(x)$ is a solution of (12.3) which is positive for $x>a$, and the variable $t$ is defined by

$$
t=\int_{b}^{x} \sigma(t) d t
$$

equation (12.2) transforms into

$$
\left(r \sigma^{3} \ddot{y}\right)^{*}+\sigma^{-1} P y=0,
$$

where dots denote differentiation with respect to $t$, and $r(x), \sigma(x), P(x), y(x)$ are taken as functions of $t$.

If $\sigma(x)$ is the solution of (12.2) determined by $\sigma(a)=0, \sigma^{\prime}(a)=1, \sigma(x)$ will be positive in $(a, \infty)$. In view of (12.4), we have

$$
\dot{y}=y^{\prime} \sigma^{-1}, \quad \ddot{y}=y^{\prime \prime} \sigma^{-2}-y^{\prime} \sigma^{\prime} \sigma^{-3},
$$


and thus

$$
\begin{aligned}
r \sigma^{3} \ddot{y} & =r \sigma y^{\prime \prime}-r y^{\prime} \sigma^{\prime}, \\
\left(r \sigma^{3} \ddot{y}\right)^{\cdot} & =\sigma^{-1}\left[\left(r \sigma y^{\prime \prime}\right)^{\prime}-\left(r y^{\prime} \sigma^{\prime}\right)^{\prime}\right] \\
& =\left(r y^{\prime \prime}\right)^{\prime}-\sigma^{-1} y^{\prime}\left(r \sigma^{\prime}\right)^{\prime} .
\end{aligned}
$$

Hence,

$$
\left(r \sigma^{3} \ddot{y}\right) \cdot \cdots+P \sigma^{-1} y=\sigma^{-1}\left\{\left(r y^{\prime \prime}\right)^{\prime \prime}-\left[\sigma^{-1} y^{\prime}\left(r \sigma^{\prime}\right)^{\prime}\right]^{\prime}+P y\right\} .
$$

Since $\sigma(x)$ is a solution of (12.3), we have $\left(r \sigma^{\prime}\right)^{\prime}=-q \sigma$, and therefore

$$
\left(r \sigma^{3} \ddot{y}\right) \cdot+P \sigma^{-1} y=\sigma^{-1}\left\{\left(r y^{\prime \prime}\right)^{\prime \prime}+\left(q y^{\prime}\right)^{\prime}+P y\right\} .
$$

In view of (12.2), $y=y[x(t)]$ is thus found to be a solution of equation (12.5).

The preceding transformation has the advantage that the coefficient of $y$ in the transformed equation (12.5) has the same sign as the coefficient of $y$ in the original equation (12.2). Many of the results derived in $\$ \S 2-11$ may therefore be immediately extended to equations (12.2) in which $P(x)$ is of constant sign, without carrying out the above transformation, providing the second-order equation (12.3) is nonoscillatory. This is true in particular of results concerning the zeros of solutions, since the transformation is easily seen to preserve the multiplicities of zeros. It may also be noted that the transformation of Theorem 12.1 is always possible if $q(x)$ is negative, since in this case equation (12.3) is trivially nonoscillatory.

The second transformation which removes the middle term of equation (12.2) is described in the following result, which is readily verified.

Theorem 12.2. Let the equation

$$
8\left(r^{1 / 2} w^{\prime}\right)^{\prime}+q r^{-1 / 2} w=0
$$

be nonoscillatory in $[a, \infty)$ and let $w(x)$ be a solution of (12.6) which is positive in $(a, \infty)$. If $y(x)$ is a solution of (12.2) and $y(x)=u(x) z(x)=w^{2}(x) z(x)$, then $z(x)$ is a solution of the equation

$$
\left(r u^{2} z^{\prime \prime}\right)^{\prime \prime}+u\left[\left(r u^{\prime \prime}\right)^{\prime \prime}+\left(q u^{\prime}\right)^{\prime}+P u\right] z=0 .
$$

In this equation the coefficient of $z(x)$ is not necessarily of the same sign as the coefficient of $y(x)$ in (12.2), and the coefficient of $z(x)$ has therefore to be computed before the theory of $\S \S 2-11$ can be applied. On the other hand, this transformation will handle cases to which the transformation of Theorem 12.1 is not applicable. This is seen particularly clearly in the case $r(x) \equiv 1$, in which the auxiliary seond-order equations (12.3) and (12.6) reduce to $\sigma^{\prime \prime}+q \sigma$ $=0$ and $8 w^{\prime \prime}+q w=0$, respectively. Since the former equation may be oscillatory while the latter is nonoscillatory, there will exist functions $q(x)$ which satisfy the hypothesis of Theorem 12.2 but not those of Theorem 12.1. 


\section{REFERENCES}

1. G. D. Birkhoff, On solutions of the ordinary linear homogeneous differential equations of the third orden Ann. of Math. vol. 12 (1911) p. 103.

2. G. Cimmino, Autosoluzioni e autovalori nelle equazioni differenziali lineari ordinarie autoaggiunte di ordine superiore, Math. Z. vol. 32 (1930) pp. 4-58.

3. R. Courant and D. Hilbert, Methods of mathematical physics, vol. 1, New York, Interscience, 1953.

4. W. B. Fite, Concerning the zeros of the solutions of certain differential equations, Trans. Amer. Math. Soc. vol. 19 (1917) pp. 341-352.

5. E. Hille, Nonoscillation theorems, Trans. Amer. Math. Soc. vol. 64 (1948) pp. 234-252. Chelsea.

6. E. Kamke, Differentialgleichungen, Lösungsmethoden und Lösungen, 3 Aufl., New York,

7. Walter Leighton, On self-adjoint differential equations of second-order, J. London Math. Soc. vol. 27 (1952) pp. 37-47.

8. R. A. Moore, The behavior of solutions of a linear differential equation of second order, Pacific J. Math. vol. 5 (1955) pp. 125-145.

9. Marston Morse, The calculus of variations in the large, Amer. Math. Soc. Colloquium Publications, vol. 18, New York, 1934.

10. Z. Nehari, Oscillation criteria for second-order linear differential equations, Trans. Amer. Math. Soc. vol. 85 (1957) pp. 428-445.

11. C. N. Reynolds, Jr., On the zeros of solutions of homogeneous linear differential equations, Trans. Amer. Math. Soc. vol. 22 (1921) pp. 220-229.

12. M. Švec, Sur les dispersions de l'équation $y^{\mathrm{iv}}+Q(x) y=0$, Chechoslovak Math. J. vol. 5 (1955) pp. 29-60.

13. W. Sternberg, Die Entwicklung unstetiger Funktionen nach den Eigenfunktionen eines eindimensionalen Randwertproblems, Math. Z. vol. 3 (1919) pp. 190-208.

14. W. M. Whyburn, On self-adjoint ordinary differential equations of the fourth order, Amer. J. Math. vol. 52 (1930) pp. 171-196.

Carnegie Institute of Technology, PitTsburgh, PA. 